\title{
Forward genetics in Wolbachia: Regulation of Wolbachia proliferation by the amplification and deletion of an addictive genomic island
}

\author{
Elves H. Duarte $\circledast^{1,2 \times a *}$, Ana Carvalho $\oplus^{1 \text { ab }}$, Sergio López-Madrigal $\oplus^{1}$, João Costa $\oplus^{1}$, \\ Luís Teixeira $\circledast^{1,3 *}$ \\ 1 Instituto Gulbenkian de Ciência, Oeiras, Portugal, 2 Faculdade de Ciências e Tecnologia, Universidade de \\ Cabo Verde, Palmarejo, Cabo Verde, 3 Faculdade de Medicina, Universidade de Lisboa, Lisboa, Portugal \\ a Current address: Department of Genetics, University of Cambridge, Cambridge, United Kingdom \\ ab Current address: Department of Evolutionary Ecology, Institute of Organismic and Molecular Evolution, \\ Johannes Gutenberg University, Mainz, Germany \\ * ehelegam@gmail.com(EHD); Iteixeira@igc.gulbenkian.pt(LT)
}

\section{Abstract}

\section{OPEn ACCESS}

Citation: Duarte EH, Carvalho A, López-Madrigal S, Costa J, Teixeira $L$ (2021) Forward genetics in Wolbachia: Regulation of Wolbachia proliferation by the amplification and deletion of an addictive genomic island. PLoS Genet 17(6): e1009612. https://doi.org/10.1371/journal.pgen.1009612

Editor: Harmit S. Malik, Fred Hutchinson Cancer Research Center, UNITED STATES

Received: May 10, 2021

Accepted: May 18, 2021

Published: June 18, 2021

Peer Review History: PLOS recognizes the benefits of transparency in the peer review process; therefore, we enable the publication of all of the content of peer review and author responses alongside final, published articles. The editorial history of this article is available here: https://doi.org/10.1371/journal.pgen.1009612

Copyright: @ 2021 Duarte et al. This is an open access article distributed under the terms of the Creative Commons Attribution License, which permits unrestricted use, distribution, and reproduction in any medium, provided the original author and source are credited.

Data Availability Statement: The authors confirm that all data underlying the findings are fully available without restriction. All numerical data and analysis files are available from the figshare
Wolbachia is one of the most prevalent bacterial endosymbionts, infecting approximately $40 \%$ of terrestrial arthropod species. Wolbachia is often a reproductive parasite but can also provide fitness benefits to its host, as, for example, protection against viral pathogens. This protective effect is currently being applied to fight arboviruses transmission by releasing Wolbachia-transinfected mosquitoes. Titre regulation is a crucial aspect of Wolbachia biology. Higher titres can lead to stronger phenotypes and fidelity of transmission but can have a higher cost to the host. Since Wolbachia is maternally transmitted, its fitness depends on host fitness, and, therefore, its cost to the host may be under selection. Understanding how Wolbachia titres are regulated and other aspects of Wolbachia biology has been hampered by the lack of genetic tools. Here we developed a forward genetic screen to identify new Wolbachia over-proliferative mutant variants. We characterized in detail two new mutants, wMelPop2 and wMelOctoless, and show that the amplification or loss of the Octomom genomic region lead to over-proliferation. These results confirm previous data and expand on the complex role of this genomic region in the control of Wolbachia proliferation. Both new mutants shorten the host lifespan and increase antiviral protection. Moreover, we show that Wolbachia proliferation rate in Drosophila melanogaster depends on the interaction between Octomom copy number, the host developmental stage, and temperature. Our analysis also suggests that the life shortening and antiviral protection phenotypes of Wolbachia are dependent on different, but related, properties of the endosymbiont; the rate of proliferation and the titres near the time of infection, respectively. We also demonstrate the feasibility of a novel and unbiased experimental approach to study Wolbachia biology, which could be further adapted to characterize other genetically intractable bacterial endosymbionts. 
(https://doi.org/10.6084/m9.figshare.14079920.

v2). Sequencing data and assembled genomes are available from BioProject: PRJNA587443 (https://

www.ncbi.nlm.nih.gov/bioproject/).

Funding: L.T. received funding for this project from the European Research Council (ERC) under the European Union's Horizon 2020 research and innovation programme (grant agreement $n^{\circ} 773260$ - WOLBAKIAN, https://erc.europa.eu) and the Fundação para a Ciência e Tecnologia (grant IF/ 00839/2015, www.fct.pt). E.H.D. was supported by the fellowship SFRH/BD/113757/2015 from

Fundação para a Ciência e Tecnologia (www.fct.pt), in the context of the Graduate Program Science for the Development. The fly work at the Fly Facility of Instituto Gulbenkian de Ciência (Oeiras, Portugal), was partially supported by the research infrastructure Congento, co-financed by Lisboa Regional Operational Programme (Lisboa2020), under the PORTUGAL 2020 Partnership Agreement, through the European Regional Development Fund (ERDF) and Fundação para a Ciência e Tecnologia (Portugal) under the project LISBOA-01-0145-FEDER-022170. The NGS analysis at the Genomics Unit of Instituto Gulbenkian de Ciência (Oeiras, Portugal), was partially supported by ONEIDA project (LISBOA-010145-FEDER-016417) co-funded by FEEI - "Fundos Europeus Estruturais e de Investimento" from "Programa Operacional Regional Lisboa 2020" and by national funds from FCT - "Fundação para a Ciência e a Tecnologia. The funders had no role in study design, data collection and analysis, decision to publish, or preparation of the manuscript.

Competing interests: The authors have declared that no competing interests exist.

\section{Author summary}

Insects often carry bacteria that live within their cells and are transmitted from the mother to the progeny. Wolbachia is one the most common of such bacteria and can strongly influence the insect biology. Its capacity to protect some hosts from viral infection is being used in the fight against mosquitoes-transmitted viruses by introducing Wolbachia in these insects. The amount of Wolbachia within the host can impact their interaction and must be well controlled. To understand this process we screened for new mutants of Wolbachia that proliferate too much in the fruit fly Drosophila melanogaster. We identified two mutants and characterized them in detail. One mutant has too many copies of a specific set of genes, confirming previous similar results. However, the other mutant Wolbachia lost those exact same genes, showing that they are particularly important in growth regulation. Moreover, we show that proliferation of different Wolbachia variants depends on temperature, and the developmental stage of the insect host. Finally, the data indicate that protection to viruses and cost of Wolbachia depend on related but different aspects of this control of growth. In summary, we show that we can screen for new mutants of Wolbachia and understand better how control of growth is genetically controlled by Wolbachia.

\section{Introduction}

Intracellular maternally-transmitted bacterial symbionts are widespread in insects [1]. These bacterial endosymbionts can be mutualistic by, for instance, complementing the diets of their hosts, and may expand the range of ecological niches of their insect hosts [1]. They can also be parasitic, often manipulating the reproduction of their hosts and promoting their spread in the host population [1]. Understanding the interaction of endosymbionts with their hosts is crucial to understand much of insect biology. A key aspect of this interaction is the regulation of endosymbiont titres, which influence the strength of the induced phenotypes and the cost to the hosts $[2,3]$.

Wolbachia is one of the most prevalent bacterial endosymbionts in arthropods, being found in approximately $40 \%$ of terrestrial arthropod species [4]. Wolbachia is broadly known as a host reproduction manipulator [5]. However, it can also be mutualistic, by, for example, providing vitamins [6] or protecting against viral pathogens $[7,8]$.

The discovery of Wolbachia-induced protection against viruses in Drosophila melanogaster, prompted its use to control arboviruses transmission by insect vectors [9]. Aedes aegypti mosquitoes trans-infected with Wolbachia have increased resistance to viruses, including dengue, chikungunya, Zika, and yellow fever viruses, and, therefore, reduced vector competence [1013]. Release of Wolbachia-carrying mosquitoes in dengue-endemic areas is likely to reduce dengue burden $[14,15]$. Despite the preliminary successful results of this strategy, we still lack knowledge on several fundamental aspects of Wolbachia biology and interaction with viral pathogens, which hinders predicting the long-term outcome of Wolbachia-based interventions to control insect-vector transmitted viruses.

Wolbachia titres are a critical factor regulating its biology and interaction with the host [3]. Titres correlate positively with transmission fidelity and the strength of Wolbachia-induced phenotypes, including the Wolbachia pathogen blocking phenotype [3,16-20]. In contrast, higher titres are associated with a reduction in host lifespan $[16,17,21,22]$. This may also have a cost to Wolbachia, since as a vertically transmitted bacterium, its fitness depends on the host fitness. Thus, Wolbachia titres regulation by the symbiont or the host may be under selection. 
Although several host and environmental factors (e.g. temperature) have been shown to affect Wolbachia titres, less is known about Wolbachia genes that regulate its titres [3].

So far, a single Wolbachia genetic factor, the Octomom region, has been shown to influence proliferation $[16,17]$. This genomic region, predicted to encode eight genes, is amplified in the highly proliferative and pathogenic $w$ MelPop. Moreover, the degree of amplification of the Octomom region determines the proliferation rate of $w$ MelPop and the strength of its life shortening phenotype [17].

The genetic intractability of Wolbachia, which remains unculturable so far, hampers the identification of more genetic modifications altering Wolbachia proliferation. Hence, unbiased approaches such as genetic screens could contribute to our understanding of the genetic bases of Wolbachia-host interactions. Here, we developed a screening strategy in Wolbachia to isolate novel over-proliferating variants. The strategy was based on random mutagenesis, which has been applied before to other unculturable bacteria [23]. We fed the mutagen ethyl methanesulfonate (EMS) to D. melanogaster females carrying Wolbachia and screened for over-proliferative Wolbachia in their progeny. This approach allowed us to isolate new over-proliferating Wolbachia mutants. We identified the genetic changes in Wolbachia causing over-proliferation and made a detailed phenotypical characterization in terms of proliferation, cost to the host, and antiviral protection. We identified a new mutation leading to Wolbachia over-proliferation and revealed a complex role for the Octomom region in regulating Wolbachia proliferation. Moreover, we demonstrated the feasibility of a novel and unbiased experimental approach to study Wolbachia biology.

\section{Results}

\section{Isolation of over-proliferative Wolbachia in an unbiased forward genetic screen}

We implemented a classical forward genetic screen in order to isolate new over-proliferative Wolbachia variants. We attempted to mutagenize Wolbachia by feeding the mutagen EMS to Wolbachia-carrying D. melanogaster females. EMS is extensively used in D. melanogaster [24] and has been previously used to mutagenize intracellular bacteria in cell culture [23]. We then tested Wolbachia titres, by real-time quantitative PCR (qPCR), in the progeny of treated females, since this bacterium is maternally transmitted. We used flies with $w$ MelCS_b as our starting variant because of its potential to easily become over-proliferative, given its genetic proximity to the over-proliferative and pathogenic $w$ MelPop variant $[16,17,22,25]$.

Putative mutagenized Wolbachia cells within the host would be in a mixed population, which would make it harder to assess their specific phenotype. However, we hypothesized that over-proliferating Wolbachia cells could overtake the population and that the resulting higher titres could be detectable. Moreover, we decided to pre-treat some of the EMS exposed females with tetracycline to reduce the Wolbachia population in these females and their progeny. This Wolbachia titre reduction should decrease competition for any new mutated Wolbachia, increase drift during vertical transmission, and, therefore, potentially facilitate fixation of new variants. To set up the conditions for tetracycline treatment, we tested different doses of this antibiotic on females, without EMS. The progeny of treated females had from 0 to $90 \%$ of the Wolbachia titres in controls (S1 Fig, $p<0.001$ for all doses compared with control, at generation 1). We then followed the subsequent progeny of these flies to test how many fly generations it takes to recover normal Wolbachia titres. Except for higher tetracycline doses which lead to infection loss, Wolbachia titres recovered to normal within four fly generations (S1 Fig, linear mixed model $[\mathrm{lmm}], p>0.48$ for all doses compared with control at generation 4 ). 
We also tested for the effect of different EMS doses on the fecundity of D. melanogaster females and Wolbachia titres. We observed that increasing doses of EMS reduce female fecundity (S2A and S2B Fig, linear model [ $1 \mathrm{~m}], p<0.001$ for both egg number and adult progeny per female). Moreover, we found that EMS feeding strongly reduces Wolbachia titres in the next generation, in a dose-dependent manner (S2C and S2D Fig, non-linear model [nls] fit, $p<0.001$ ). Titres were reduced by up to $90 \%$ when $8,000 \mathrm{mM}$ EMS was supplied, leading to the loss of Wolbachia in the next generation in some lines (S2C and S2D Fig). Given these results and the recovery time after tetracycline treatment detailed above, we quantified Wolbachia titres at the first generation (F1), the immediate progeny of EMS-treated females, and at the fourth generation after treatment (F4), when we would expect Wolbachia titres to recover after the severe reduction due to EMS treatment.

We screened approximately one thousand F1 progeny of EMS-treated females, in a range of experimental conditions, and at least one $\mathrm{F} 4$ female descendent per treated female. We varied EMS dose from $10 \mathrm{mM}$ to $8,000 \mathrm{mM}$, and tetracycline dose from $0 \mu \mathrm{g} / \mathrm{ml}$ to $12.5 \mu \mathrm{g} / \mathrm{ml}$, in different combinations (S1 Table). The relative Wolbachia titre was determined when females were ten days old, after they laid eggs, so that any putatively interesting progeny could be followed up.

In three independent batches of EMS-treated flies, we detected females with 3 to 14-fold more Wolbachia than controls, suggesting the presence of over-proliferative variants (Fig 1 and S3 Fig). In two batches, over-proliferating Wolbachia were identified in the F1 and in the other batch in the F4. We assessed Wolbachia titres in the next generation and found that the over-proliferative phenotypes were inherited. Subsequent selection allowed us to establish $D$. melanogaster lines carrying new potentially over-proliferative Wolbachia variants.

We designed the screen to find new mutants of Wolbachia that lead to the endosymbiont over-proliferation. However, EMS will most likely also induce mutations in the host, in the nuclear or mitochondrial genomes, that can be transmitted. To minimize the influence of host nuclear mutations on our screen, we backcrossed the EMS-treated females and their progeny, at every generation, with males from the control isogenic line. To verify that new mutations in

A

Relative Wolbachia titre

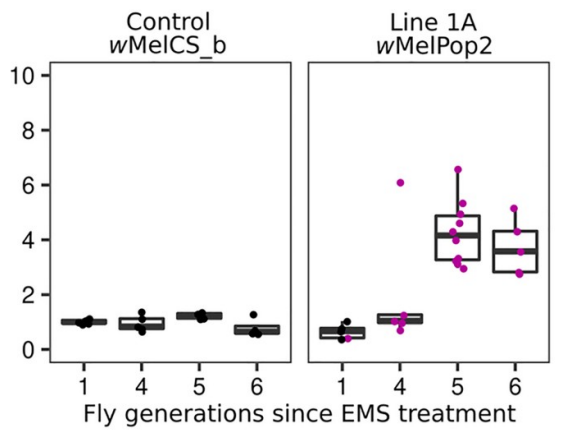

B

Relative Wolbachia titre

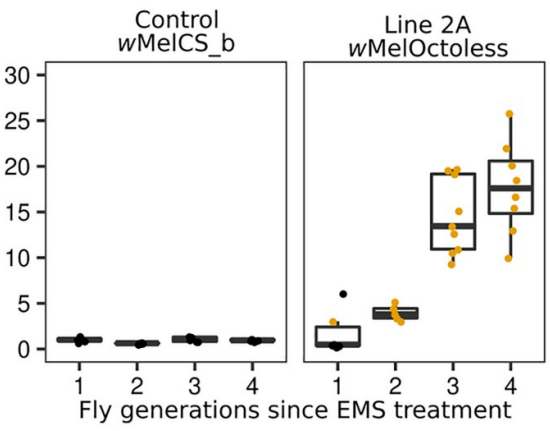

C Relative Wolbachia titre

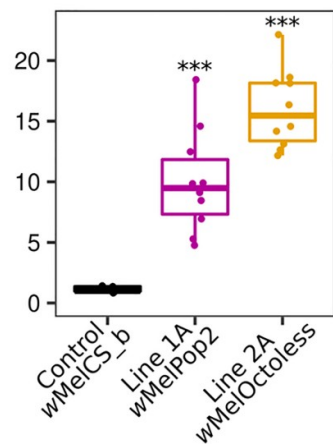

Fig 1. Isolation of over-proliferative Wolbachia variants by a forward genetic screen. (A and B) Relative Wolbachia titres in a control (wMelCS_b) and EMS-treated flies (Lines 1A and 2A). 5-10 virgin females were randomly collected each generation for egg-laying and Wolbachia titre measurement using qPCR. Bacterial titres are normalized to that of control flies. The female used in the first generation to start the next generation is coloured. At the other generations the progeny of the female with the higher Wolbachia titre was used to set up the next generation. The selection of the other putative over-proliferating Wolbachia line in panel B is shown in S3A Fig. (C) Relative titres of over-proliferating Wolbachia variants in a host isogenic genetic background. Both lines kept the over-proliferative phenotype $(p<0.001)$. Each dot represents the Wolbachia titre of a single female.

https://doi.org/10.1371/journal.pgen.1009612.g001 
the host nuclear genome were not the cause of Wolbachia over-proliferation, we replaced the first, second and third chromosomes of D. melanogaster females carrying the over-proliferating Wolbachia variants in lines $1 \mathrm{~A}, 2 \mathrm{~A}$, and $3 \mathrm{~A}$, with the chromosomes of the control line, through the use of balancer chromosomes (S4 Fig). We then repeated Wolbachia titres quantification and found that the over-proliferative phenotypes were maintained (Figs $1 \mathrm{C}$ and S5, $\operatorname{lmm}, p<0.001$ for all compared with $w$ MelCS_b).

Since mitochondria are maternally transmitted and could have been also mutated by EMS, the experiments described above cannot exclude the possibility that Wolbachia over-proliferation is mitochondria-determined. Thus, the mitogenome of the lines $1 \mathrm{~A}$ and $2 \mathrm{~A}$, showing higher Wolbachia titres, were sequenced with Illumina short-reads and aligned to the mitochondrial reference genome release 6 (GenBank: KJ947872.2:1-14,000, S2 Table). We did not find SNPs or indels unique to the mitochondria of these flies, which shows that flies with overproliferative Wolbachia did not inherit mutated mitochondria (S3 Table). Therefore, we concluded that the observed Wolbachia over-proliferative phenotypes did not result from mutations in neither the nuclear or mitochondrial host genome.

\section{Identification of genetic basis of the new over-proliferative variants}

To identify the mutations associated with over-proliferation, we sequenced and assembled the genomes of these Wolbachia variants. We performed a hybrid assembly with short (Illumina) and long-reads (Nanopore) and obtained single and circular genomes for each Wolbachia chromosome (S2 and $\mathrm{S} 4$ Tables).

To test our assembly pipeline we sequenced and assembled a previously characterized Clade III $w$ Mel Wolbachia variant, named $w \mathrm{Mel}[16]$, which derives from the line used for the original $w$ Mel reference genome (GenBank: AE017196.1) [26]. The new $w$ Mel genome (GenBank: CP046925.1) was also circular and comparable in size, structure and number of ORFs with previously published $w$ Mel genomes [26,27], including the $w$ Mel reference genome (S4 Table). We found, however, two SNPs and seven indels relative to this reference genome, which we confirmed using Sanger sequencing (S5 Table). These results validate our sequencing pipeline.

We assembled the genome of the control variant $w$ MelCS_b using this pipeline (GenBank: CP046924.1), in order to be able to identify new mutations in the new variants. We also compared this new assembly of $w$ MelCS_b with the $w$ Mel reference genome (GenBank: AE017196.1) and identified 37 indels and 146 SNPs between these variants (S6 Table).

The only difference between the genome of the over-proliferative Wolbachia variant in Line 1A (GenBank: CP046922.1) and wMelCS_b was an amplification of the Octomom region (Fig $2 \mathrm{~A}$ and $\mathrm{S} 1 \mathrm{Text}$ ). There were three more copies of this region, giving a genome size difference of $62,814 \mathrm{bp}$. The Octomom region amplification, and lack of other differences, was also confirmed by mapping of the Illumina sequencing reads from Line $1 \mathrm{~A}$ on the genome of wMelCS_b (GenBank: CP046924.1) and by qPCR (Figs 2B and S6). These results show that Octomom amplification is the cause of over-proliferation, consistently with previous findings with the variant $w$ MelPop $[16,17,28]$. As shown before for $w$ MelPop [17], we observed variation in the Octomom copy number in wMelPop2-carrying flies. For further analyses of the phenotype of this variant we established, through selection (as in [17]), D. melanogaster lines carrying Wolbachia with low (2-3) or high Octomom (8-9) copy number (S7 Fig). We named this variant $w$ MelPop2, given the nature of the genomic change inducing its overproliferation.

We sequenced and assembled the $w$ MelPop genome following the same pipeline (GenBank: CP046921.1) and compared it to wMelPop2. We only detected the two SNPs previously 
$\mathbf{A}$

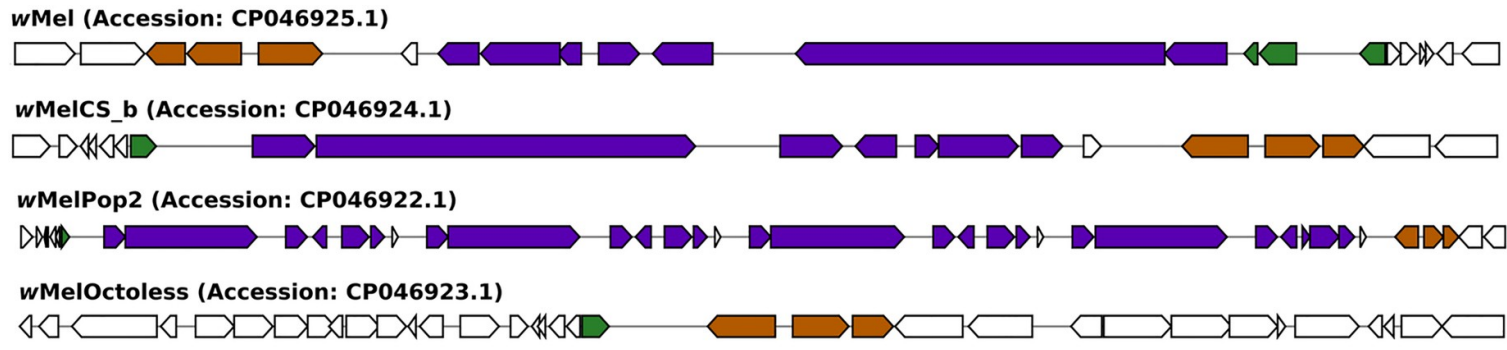

B

Relative coverage
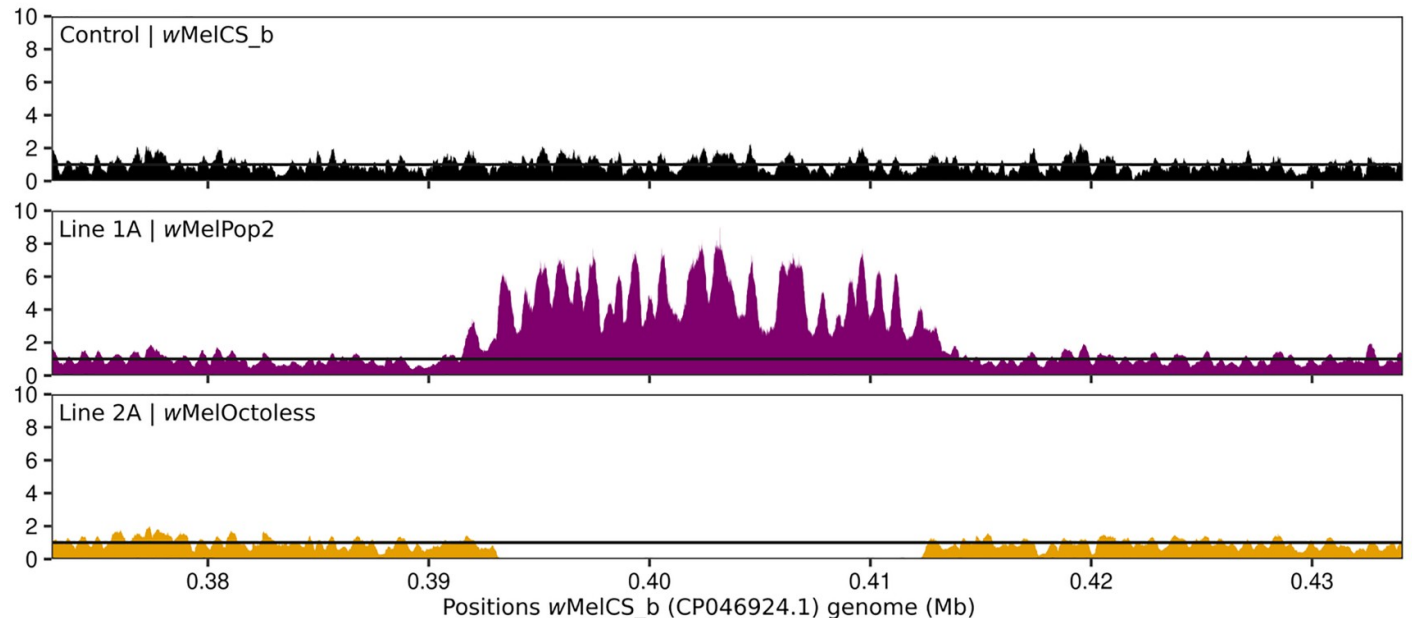

Fig 2. Both amplification and deletion of Octomom lead to Wolbachia over-proliferation. (A) Representation of Octomom region and its flanking region in over-proliferative Wolbachia. De novo assembled genomes of $w \mathrm{Mel}, w \mathrm{MelCS} \_\mathrm{b}$, Line1A (wMelPop2) and Line 2A ( $w$ MelOctoless) were annotated using the NCBI Prokaryotic Genome Annotation Pipeline v4.10. These representations were generated using MultiGeneBlast v1.1.13 (http://multigeneblast.sourceforge.net/) and identify the homologous genes immediately upstream of Octomom (orange), in the Octomom region (purple), and immediately downstream of Octomom (green), in the $w$ Mel reference genome (GenBank: AE017196.1). Regions are not to scale. Note that the genome annotation differs between this new $w \mathrm{Mel}$ genome assembly and the reference genome, although there is no difference between the sequences in this region. (B) Relative coverage in the genomic region containing the Octomom region. Illumina paired-end reads of the different Wolbachia variants were mapped to wMelCS_b genome (GenBank: CP046924.1).

https://doi.org/10.1371/journal.pgen.1009612.g002

identified between $w$ MelCS_b and $w$ MelPop (position 920,191: T in $w$ MelPop and C in $w$ MelPop2; and position 1,005,339: A in wMelPop and G in wMelPop2 [16]). We also compared the mitogenome of flies carrying $w$ MelPop and $w$ MelPop2 and found one single substitution (position 10,793: G in wMelPop and A in wMelPop2) (S3 Table), which we confirmed using Sanger sequencing. Genome assembly and individual Nanopore long-reads from $w$ MelPop and $w$ MelPop2 (S7 Table, S8 Fig) show that Octomom amplification in these variants occurs in tandem, as previous data indicated [17].

Interestingly, the genome of the over-proliferative Wolbachia variant in line 2A (GenBank: CP046923.1) only differs from $w$ MelCS_b by a deletion of a 20,938bp genomic fragment that includes the full-length Octomom region and one of its flaking direct repeats (Fig 2A and S1 Text). Mapping the Illumina sequencing reads of this variant on the genome of $w$ MelCS_b (GenBank: CP046924.1) confirmed this deletion as the only difference between the two (Fig 2B). The absence of all Octomom genes in this line was also confirmed by qPCR (S6 Fig). 
These results identify loss of the Octomom region as the cause of this variant over-proliferative phenotype. Thus, we named this variant $w$ MelOctoless.

The variant in line $2 \mathrm{~B}$, isolated together with $w$ MelOctoless, also lost the Octomom region. This was the only observed difference when mapping the Illumina reads on $w$ MelCS_b (S9 Fig), and no differences were observed when the Illumina reads were mapped to the $w$ MelOctoless genome (GenBank: CP046923.1). Since wMelOctoless and the variant in line 2B were identified in the same batch of mutagenesis, they may not be independent. However, and importantly, we obtained the same results with another independent over-proliferative line, isolated in a different batch of treatment, line 3A (S3 and S9 Fig). Mapping the Illumina sequence reads from this line to $w \mathrm{MelCS} \_\mathrm{b}$ also identifies the loss of Octomom as the only mutation in this variant. Accordingly, there are no differences to $w$ MelOctoless. Therefore, we named this line $w$ MelOctoless 2 . These results further confirm that loss of the Octomom region leads to an over-proliferative phenotype in Wolbachia.

In summary, we were able to identify the genomic changes associated with the new overproliferative variants and all map to loss or amplification of the Octomom region.

\section{Deletion and amplification of the Octomom region differently impact titres and growth of Wolbachia}

In order to characterize better the phenotypes of the new Wolbachia variants wMelOctoless and $w$ MelPop2, we analysed their proliferation, together with $w$ MelCS_b and $w$ MelPop, in adult males kept at $18^{\circ} \mathrm{C}, 25^{\circ} \mathrm{C}$, and $29^{\circ} \mathrm{C}$ (Fig 3 and $\mathrm{S} 10 \mathrm{Fig}$ ). The flies were reared at $25^{\circ} \mathrm{C}$ and placed at the different temperatures when $0-1$ day-old adults. At this initial point, at adult eclosion, there are differences in titres between lines carrying different Wolbachia variants (S11 Fig, $p<0.028$ for all comparisons). Flies carrying $w$ MelCS_b have the lowest relative titre of Wolbachia. Flies carrying variants with low amplification of the Octomom region have approximately twice the titres of Wolbachia, while flies carrying variants with high copy number of this region have three times more Wolbachia than wMelCS_b. Finally, flies carrying $w$ MelOctoless have the highest titres, approximately four-fold higher than flies carrying $w$ MelCS_b. Therefore, the deletion or amplification of the Octomom region impact Wolbachia titres at adult eclosion.

To analyse proliferation during adult life, we fitted an exponential model to the titres over adult age and estimated doubling time of the Wolbachia variants, at different temperatures (Table 1). Doubling time varies widely with Wolbachia variant and temperature, from approximately one day to seventeen days. A model with all the data shows a complex interaction between proliferation, Wolbachia variant and temperature $(\mathrm{lmm}, p<0.001)$. We analysed this dataset by comparing specific set of variants to test differences between $w$ MelOctoless and $w$ MelCS_b, differences between $w$ MelPop2 and wMelCS_b, and differences between levels of Octomom amplification in $w$ MelPop and $w$ MelPop2.

A direct comparison between $w$ MelOctoless with $w$ MelCS_b shows that this new variant replicates faster than $w$ MelCS_b $(\mathrm{lmm}, p<0.001)$, although it is a relatively small difference at all temperatures (in the full model with all variants, however, the proliferation of $w$ MelOctoless and $w$ MelCS_b is only statistically different at $18^{\circ} \mathrm{C}$, Table 1 ). Both strains interact equally with temperature. Their growth rate does not significantly change between $18^{\circ} \mathrm{C}$ and $25^{\circ} \mathrm{C}$ $(p=0.94)$, but increases at $29^{\circ} \mathrm{C}(p<0.001)$.

A comparison of $w$ MelPop2 having high and low Octomom copy number with $w$ MelCS_b and $w$ MelOctoless shows that these variants with Octomom amplification have the highest growth rates at $25^{\circ} \mathrm{C}$ and $29^{\circ} \mathrm{C}(p<0.001$ for all comparisons of $w$ MelPop2 ( 3 or $8-9$ copies) compared to $w$ MelCS_b and $w$ MelOctoless). At $18^{\circ} \mathrm{C} w$ MelPop2 with 3 copies of Octomom 
A

Relative Wolbachia titre at $18^{\circ} \mathrm{C}$

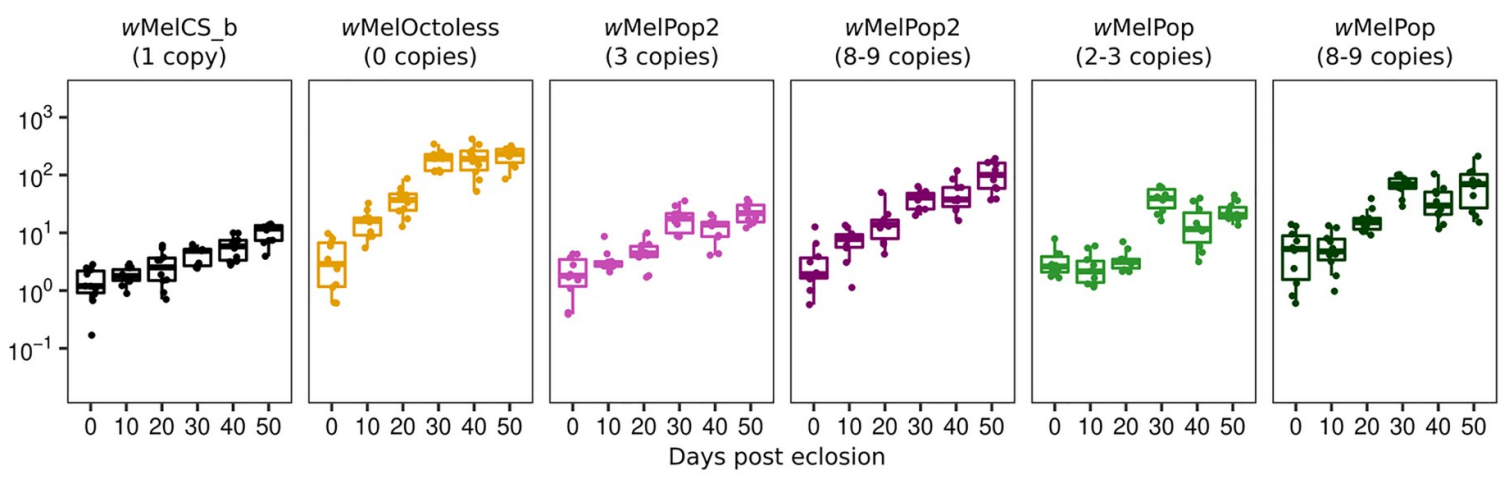

B

Relative Wolbachia titre at $25^{\circ} \mathrm{C}$

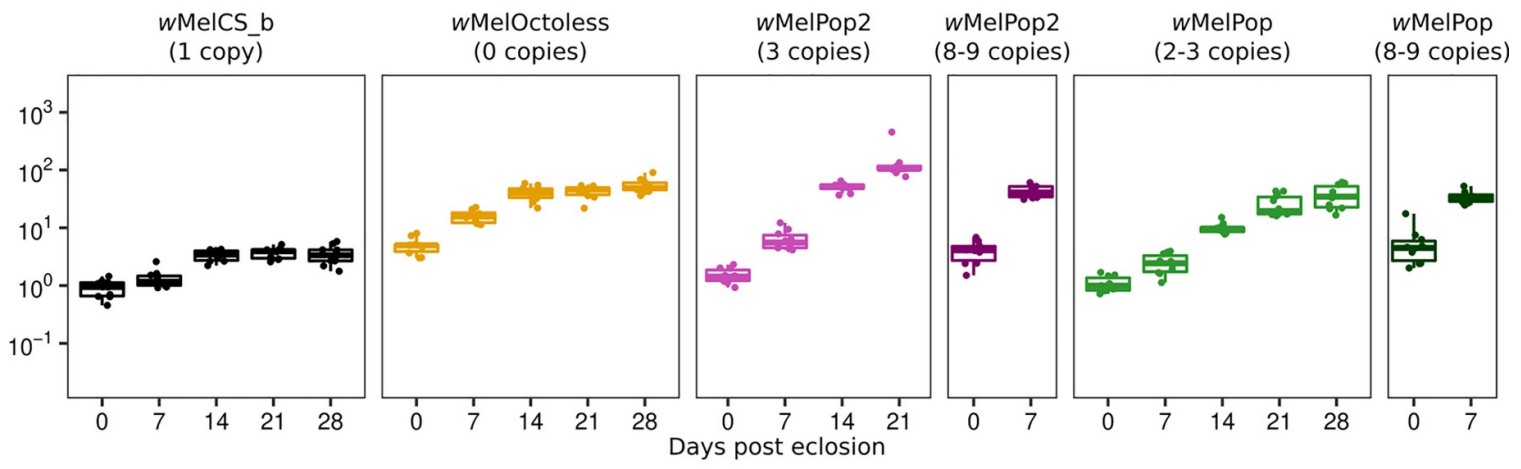

C

Relative Wolbachia titre at $29^{\circ} \mathrm{C}$
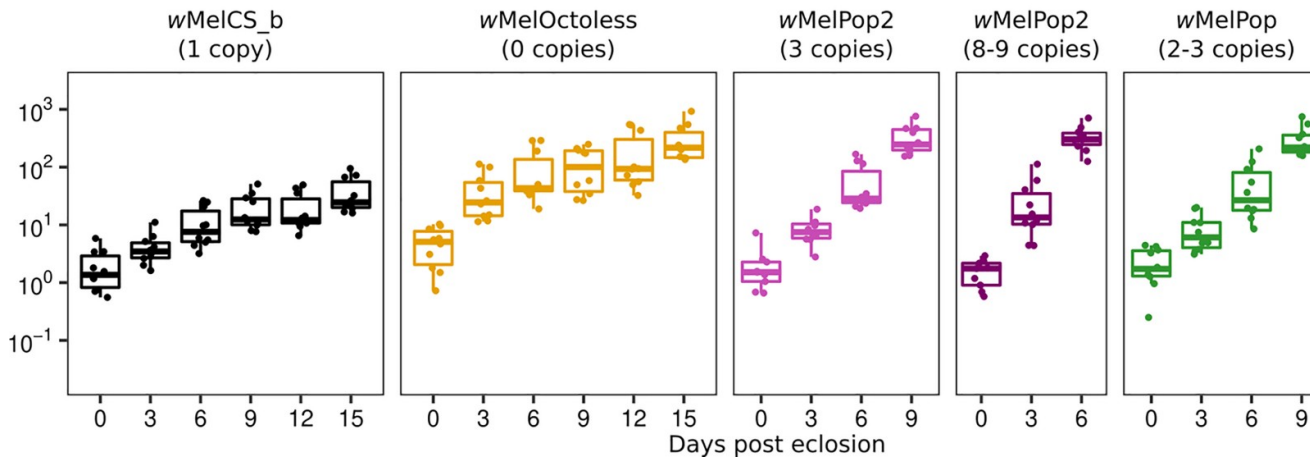

wMelPop

Fig 3. The amplification or deletion of Octomom increase Wolbachia proliferation rate in adults. Time-course of relative Wolbachia titres in adults at $18^{\circ} \mathrm{C}(\mathrm{A}), 25^{\circ} \mathrm{C}(\mathrm{B})$ and $29^{\circ} \mathrm{C}(\mathrm{C})$ with different Wolbachia variants. D. melanogaster males used in these experiments developed at $25^{\circ} \mathrm{C}$, were collected on the day of adult eclosion and aged at the given temperatures $\left(18^{\circ} \mathrm{C}, 25^{\circ} \mathrm{C}\right.$ or $\left.29^{\circ} \mathrm{C}\right)$. Ten males were collected at each time-point for Wolbachia titre measurement using qPCR. Wolbachia titres were normalized to that of $0-1$ day-old $w$ MelCS_b-infected males. A replicate of the experiment is shown in S10 Fig. Exponential models were used to estimate Wolbachia doubling time, using both full replicates, and a summary of the results is given in Table 1. Each dot represents the relative Wolbachia titre of a single male.

https://doi.org/10.1371/journal.pgen.1009612.g003

has a growth rate similar to $w$ MelCS_b $(p=0.79)$ and lower than $w$ MelOctoless $(p<0.001)$. While at this temperature the growth rate of $w$ MelPop2 with 8-9 copies of Octomom is not significantly different from either $w$ MelCS_b or $w$ MelOctoless $(p>0.088$ in both comparisons), and the estimated value is in-between the two (Table 1). The analysis also shows a strong 
Table 1. Doubling time of Wolbachia variants in larvae and adults at different temperatures.

\begin{tabular}{|c|c|c|c|c|}
\hline & \multicolumn{4}{|c|}{ Doubling time-days (95\% confidence interval) } \\
\hline & \multirow{2}{*}{$\begin{array}{c}\text { Larvae } \\
25^{\circ} \mathrm{C}\end{array}$} & \multicolumn{3}{|c|}{ Adults } \\
\hline & & $18^{\circ} \mathrm{C}$ & $25^{\circ} \mathrm{C}$ & $29^{\circ} \mathrm{C}$ \\
\hline \multirow[t]{2}{*}{$w$ MelCS_b } & 0.68 & 16.79 & 13.90 & 4.07 \\
\hline & $(0.55-0.91)$ & $(13.29-22.79)$ & $(9.84-23.62)$ & $(3.35-5.17)$ \\
\hline \multirow[t]{2}{*}{$w$ MelOctoless } & 0.50 & 9.41 & 10.46 & 3.37 \\
\hline & $(0.42-0.61)$ & $(8.20-11.04)$ & $(7.99-15.16)$ & $(2.86-4.10)$ \\
\hline \multirow[t]{2}{*}{$w$ MelPop2 (3 copies) } & n.d. & 17.68 & 3.50 & 1.28 \\
\hline & & $(13.84-24.45)$ & $(3.02-4.15)$ & $(1.13-1.48)$ \\
\hline \multirow[t]{2}{*}{$w$ MelPop2 (8-9 copies) } & 0.59 & 11.86 & 2.26 & 1.07 \\
\hline & $(0.48-0.75)$ & $(10.00-14.56)$ & $(1.74-3.22)$ & $(0.92-1.28)$ \\
\hline \multirow[t]{2}{*}{$w$ MelPop ( $2-3$ copies $)$} & n.d. & 15.44 & 5.30 & 1.38 \\
\hline & & $(12.43-20.36)$ & $(4.50-6.44)$ & $(1.22-1.60)$ \\
\hline \multirow[t]{2}{*}{$w$ MelPop (8-9 copies) } & n.d. & 13.82 & 2.79 & 0.88 \\
\hline & & $(11.33-17.70)$ & $(2.04-4.43)$ & $(0.76-1.02)$ \\
\hline
\end{tabular}

n.d.-not determined

https://doi.org/10.1371/journal.pgen.1009612.t001

interaction between $w$ MelPop2 growth and temperature. Both low and high Octomom copy number $w$ MelPop2 growth rates increase from $18^{\circ} \mathrm{C}$ to $25^{\circ} \mathrm{C}$, and from $25^{\circ} \mathrm{C}$ to $29^{\circ} \mathrm{C}$ ( $p<0.001$ for these comparisons).

To test the effect of the degree of Octomom amplification on growth rate and differences between $w$ MelPop and $w$ MelPop2, we compared these variants with low or high copy number of the Octomom region. The variants with high copy number have a higher growth rate than the variants with low copy number at all temperatures ( $p<0.025$ at all temperatures). These results confirm that the degree of amplification of the Octomom region controls the intensity of the over-proliferation of these variants, as shown before [17]. Both low and high Octomom copy number $w$ MelPop and $w$ MelPop2 increase growth rate with temperature $(p<0.001$ for low and high copy number variants compared between $18^{\circ} \mathrm{C}$ and $25^{\circ} \mathrm{C}$, and between $25^{\circ} \mathrm{C}$ and $29^{\circ} \mathrm{C}$ ), confirming the analysis above.

The statistical model comparing $w$ MelPop and $w$ MelPop2, which differ in two SNPs (see above), indicated a significant difference in growth between them at $25^{\circ} \mathrm{C}(p<0.001)$. This could mean that these two SNPs also influence growth of Wolbachia. However, this could also be due to the fact that the copy number of the Octomom region was not equally controlled in $w$ MelPop and $w$ MelPop2 lines during these experiments. $w$ MelPop low copy number line had $2-3$ copies of Octomom, while the $w$ MelPop2 line had 3 copies. To test if $w$ MelPop and $w$ MelPop2 indeed vary in proliferation rate, we repeated this experiment with a more tightly controlled Octomom copy number in these two lines, at $25^{\circ} \mathrm{C}$ (S12A and S12B Fig). Both $w$ MelPop and $w$ MelPop 2 carrying 3 copies of Octomom grow faster than $w$ MelCS_b (lmm, $p<0.001$ for both $)$ and there is no difference in growth between them $(p=0.39)$. This indicates that the genetic differences between these lines do not affect their growth and that they are equally influenced by Octomom copy number.

Overall, the data and analysis show a complex interaction between Wolbachia variants, temperature and growth rate. There is a strong interaction between temperature and the increased proliferation of variants with amplification of the Octomom region, $w$ MelPop and $w$ MelPop2, when compared with $w$ MelCS_b. The effect of the amplification is not significant at $18^{\circ} \mathrm{C}$ and becomes increasingly stronger at higher temperatures. On the other hand, loss of Octomom 
Wolbachia genome copies

uMeICS b

(1 copy)

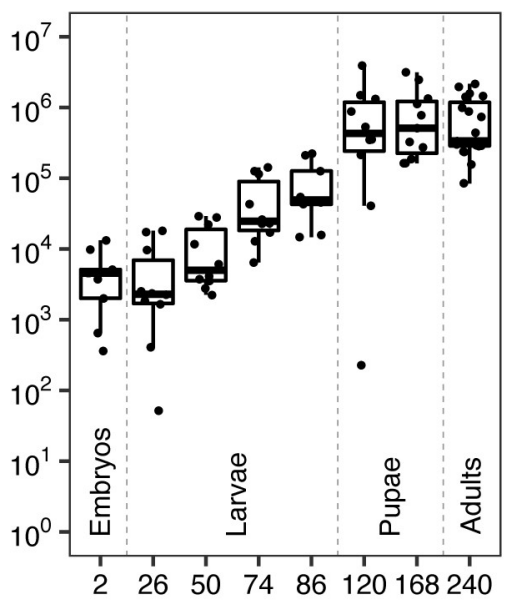

wMelOctoless

(0 copies)

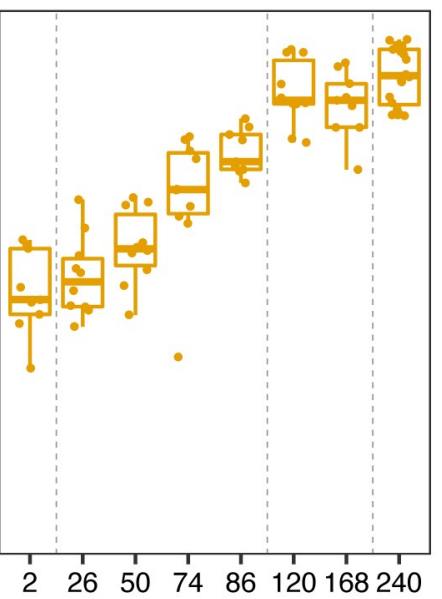

Hours post egg-laying
wMelPop2

(8-9 copies)

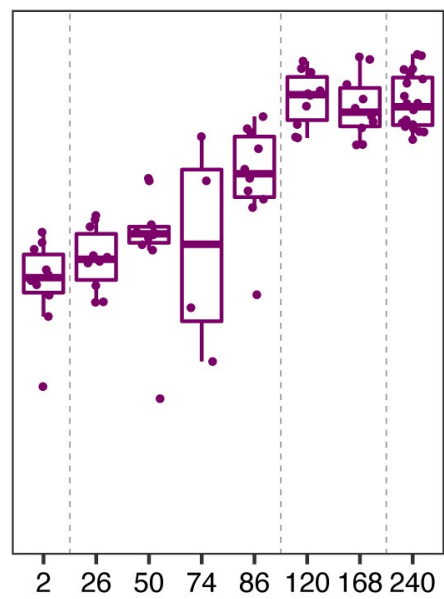

Fig 4. Wolbachia proliferates rapidly during larval development. Wolbachia genome copies throughout D. melanogaster development. Samples are embryos (2h), $1^{\text {st }}$ instar larvae (26h), $2^{\text {nd }}$ instar larvae (50h), $3^{\text {rd }}$ instar larvae (74h and $86 \mathrm{~h}$ ), white prepupae (120h), P8 staged pupae (168h), and newly eclosed adults (240h). Vertical dashed lines separate developmental stages (i.e. eggs, larvae, pupae, and adults). Dots represent either a pool of 10 individuals (embryos and larvae) or a single individual (pupae and adults). The $\mathrm{x}$-axis is not in a continuous scale. Proliferation of the different Wolbachia variants in the first 120 hours was analysed using an exponential model. A summary is given in Table 1.

https://doi.org/10.1371/journal.pgen.1009612.g004

leads to a smaller effect in growth, but similar at all temperatures, when compared with $w$ MelCS_b. Therefore, although both genomic mutations lead to an increase in Wolbachia titres they have different impacts in the growth rates and interaction with temperature.

\section{Rapid proliferation of Wolbachia during the host development}

We also analysed the growth of $w$ MelCS_b, wMelOctoless and $w$ MelPop2 (8-9 Octomom copies) during host development. D. melanogaster develops from egg to adult in only 10 days, at $25^{\circ} \mathrm{C}$. We predicted that Wolbachia would grow much faster during this period than during adult life, considering changes in Wolbachia loads from eggs to adults [29]. We, therefore, estimated absolute numbers of Wolbachia genome copies in individuals at the different stages of development using qPCR for the single copy gene $w s p$ and a calibration curve using a plasmid with $w s p$ cloned. Assuming one chromosome per Wolbachia cell [30], these numbers

Table 2. Wolbachia genome copies in embryos and newly eclosed adult flies.

\begin{tabular}{l|c|c|c}
\hline & \multicolumn{3}{|c}{ Genome copy number (95\% confidence interval) } \\
\hline & \multicolumn{3}{|c}{ Wolbachia variants } \\
\hline Embryos & wMelCS_b & $w$ MelOctoless & $(1.280-7,380)$ \\
\hline Adults & 3,070 & 2,310 & \\
\hline Males & $(1,220-7,730)$ & $(920-5,800)$ & \\
\hline Females & & & 826,000 \\
\hline & $(222,000-597,000)$ & $2,083,000$ & $(512,000-1,334,000)$ \\
\hline
\end{tabular}

https://doi.org/10.1371/journal.pgen.1009612.t002 
correspond to Wolbachia cells. Embryos with 0-2h have between 2,300 and 3,100 Wolbachia genome copies, with no significant difference between Wolbachia variants $(\operatorname{lm}, p=0.87$ for the effect of Wolbachia variant, Fig 4, Table 2). At the end of development, newly eclosed adults carry from approximately 400,000 to 3,200,000 Wolbachia genome copies. At this stage, however, and as observed above (S11 Fig), there are significant differences between the three variants $(\operatorname{lm}, p<0.008$ for all comparisons, Table 2, Fig 4). Also, males carry less Wolbachia than females $(p=0.033)$.

Wolbachia growth seems to be restricted to the period between egg and white prepupae $(120 \mathrm{~h})$, since there is no significant growth from this stage to adults $(\mathrm{lm}, p=0.46)$. From eggs to white prepupae there is rapid exponential growth of all variants (Fig 4, Table 1). wMelCS_b has an estimated doubling time of approximately $16 \mathrm{~h}, w \mathrm{MelPop} 2$ of $14 \mathrm{~h}$, and $w$ MelOctoless of 12h. These different doubling times probably explain how Wolbachia variants reach different amounts per individual host in adults, starting from the same estimated amount in embryos. However, in this analysis the difference between growth rates is not statistically significant ( $p=0.12$ for interaction between Wolbachia variants and growth). The growth rates of these variants are, therefore, very similar during this stage, and much faster than in adults. At the same temperature, we estimated doubling times in adults of $w$ MelCS_b, $w$ MelOctoless, and $w$ MelPop2 (high-copy) to be, approximately, 13.9, 10.5, and 2.3 days, respectively (Table 1). Therefore, Wolbachia growth at different stages of D. melanogaster can vary dramatically, and the different variants respond differently to different stages of the host life cycle.

We also asked if Wolbachia Octomom copy number changed in $w$ MelCS_b and $w$ MelPop2, throughout development, as Wolbachia is proliferating fast, and found no evidence of so $(\mathrm{lm}$, $p=0.49$, S13A Fig). However, during adult life there was a small increase of Octomom copy number with age in $w$ MelPop and $w$ MelPop2 (an increase of 0.032 per day, lmer, $p=0.009$, S13B Fig), as shown before [31].

\section{Wolbachia variants with a deletion or amplification of the Octomom region induce different life-shortening phenotypes}

The over-proliferation of $w$ MelPop has been associated with a shortening of the host lifespan $[16,22]$. We, therefore, tested if these new over-proliferative variants also shorten the lifespan of their host, at different temperatures, in males (Figs 5A-5D and S14A-S14C). We also performed this assay in females at $25^{\circ} \mathrm{C}$, with similar results to males at $25^{\circ} \mathrm{C}(\mathrm{S} 14 \mathrm{D}$ and S14E Fig). There was a significant interaction between Wolbachia variant and temperature (Cox proportional hazard model with mixed effects $(\mathrm{CHR}), p<0.001)$. All lines, including the Wolbachia-free line have a shorter lifespan at $25^{\circ} \mathrm{C}$ than at $18^{\circ} \mathrm{C}$, and even shorter at $29^{\circ} \mathrm{C}$ $(p<0.001$ for all these comparisons). $w$ MelCS_b did not affect the host lifespan at any temperature ( $p>0.16$ for all comparisons with the Wolbachia-free line).

$w$ MelOctoless strongly reduces host lifespan at all tested temperatures $(p<0.001$, each comparison with wMelCS_b) (Figs 5A-5D and S14). This deleterious effect is stronger at $18^{\circ} \mathrm{C}$, where $w$ MelOctoless is the tested variant with the highest impact on lifespan, although very similar and not statistically different from $w$ MelPop2 with high Octomom copy number $(p<0.001$, for all comparisons with other lines, $p=1$ when compared with $w$ MelPop 2 with $8-9$ Octomom copies). The effect of $w$ MelOctoless on host lifespan is weaker at $25^{\circ} \mathrm{C}$ than at $18^{\circ} \mathrm{C}(p=0.001)$, and similar at $25^{\circ} \mathrm{C}$ and $29^{\circ} \mathrm{C}(p=0.95)$. These results demonstrate that the new over-proliferative $w$ MelOctoless also has a cost to the host in terms of lifespan and this effect interacts with temperature, being stronger at lower temperature.

$w$ MelPop2, similarly to $w$ MelPop, also shortens host lifespan (Figs 5 and S14). The variants containing high copy number of Octomom (8-9 copies) shorten lifespan at all temperatures 
A

Percentage of males alive at $18^{\circ} \mathrm{C}$

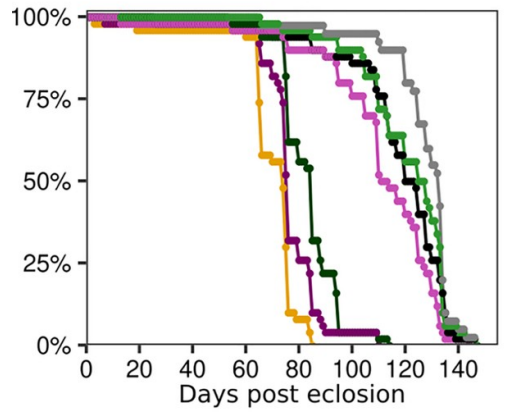

B

Percentage of males alive at $25^{\circ} \mathrm{C}$

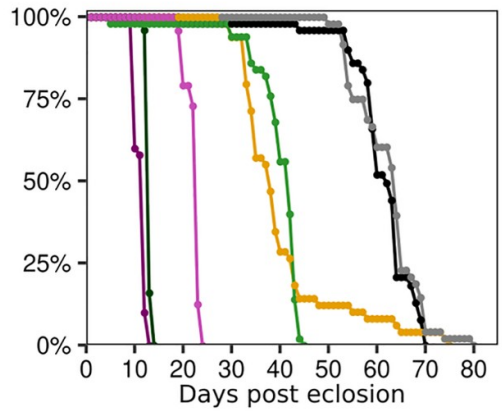

C

Percentage of males alive at $29^{\circ} \mathrm{C}$

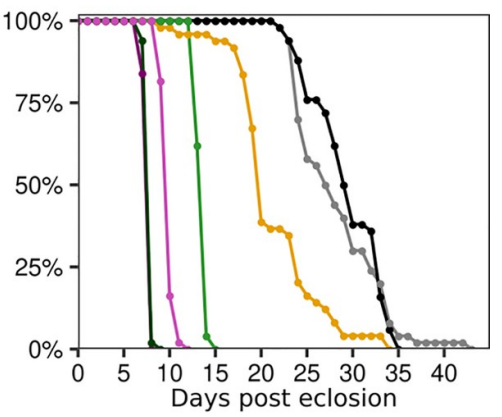

$\because$ Wolbachia-free $\rightarrow$ wMelCS_b (1 copy) $\quad \rightarrow$ wMelPop2 (3 copies) $\rightarrow$ wMelPop (2-3 copies)

D

Coefficients of Cox mixed model
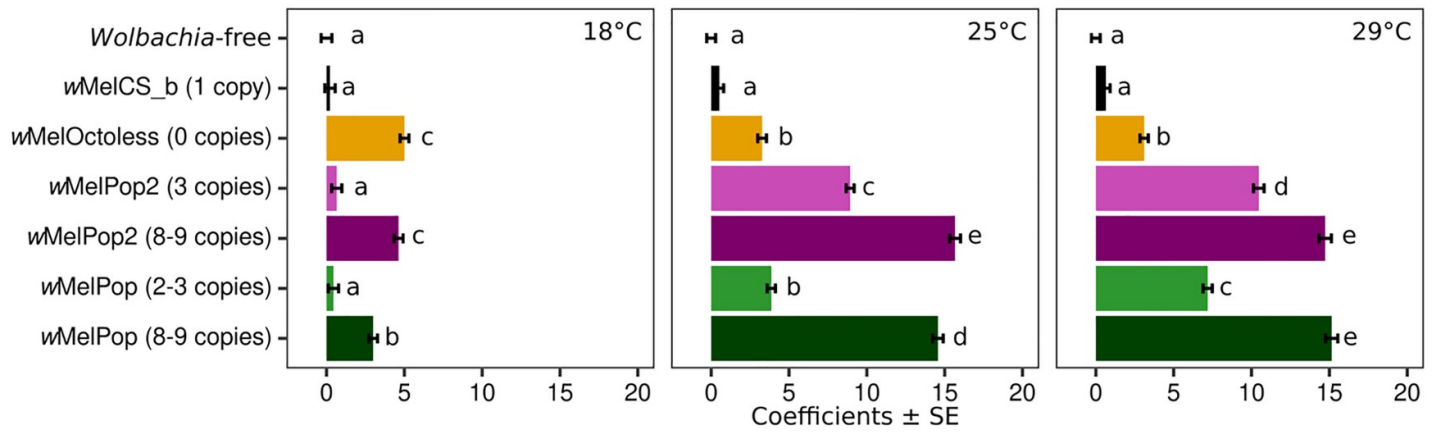

E

Life-shortening vs. doubling time $18^{\circ} \mathrm{C}$

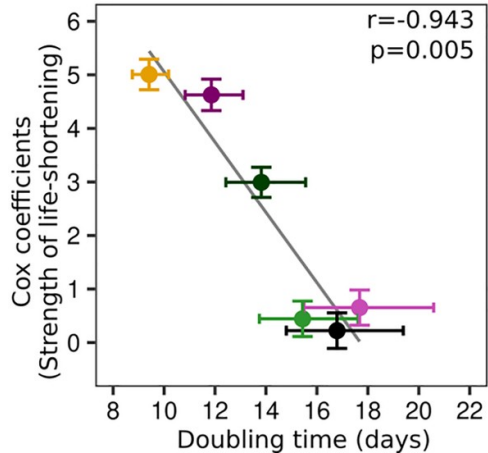

F

Life-shortening vs. doubling time $25^{\circ} \mathrm{C}$

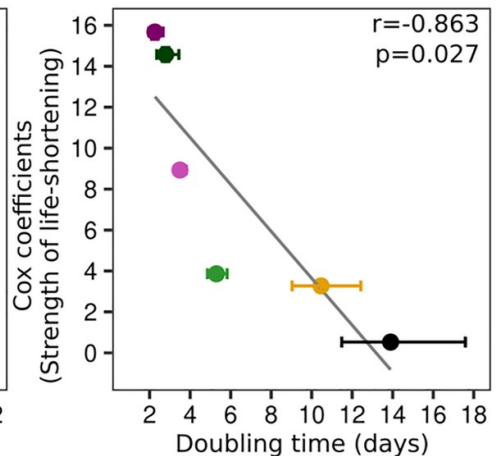

G

Life-shortening vs. doubling time $29^{\circ} \mathrm{C}$

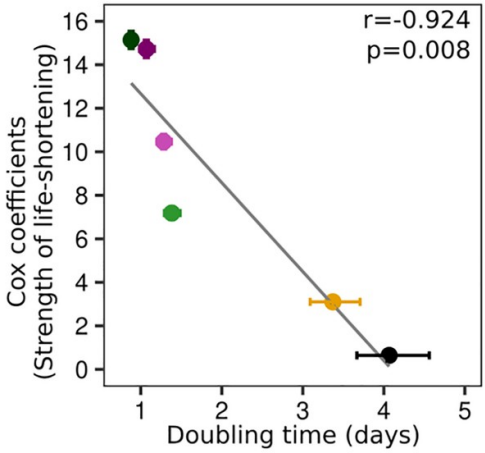

Fig 5. $w$ MelOctoless and $w$ MelPop 2 are pathogenic. Lifespan of males with different Wolbachia variants at $18^{\circ} \mathrm{C}(\mathrm{A}), 25^{\circ} \mathrm{C}(\mathrm{B})$, and $29^{\circ} \mathrm{C}(\mathrm{C})$. For survival analyses, fifty males were collected on the day of eclosion and kept in groups of 10 per vial until all flies died. Flies were transferred to new vials every five days. A full replicate of these experiments is shown in S14A-S14C Fig. (D) Coefficients of a Cox mixed model, which represent the effect of Wolbachia on the lifespan of flies relative to the lifespan of Wolbachia-free flies. Both experimental replicates were analysed together. Bars represent the standard error of the coefficient, and letters statistically significant groups after $p$-value correction. (E-G) Correlation between the strength of life-shortening phenotype and Wolbachia doubling time at $18^{\circ} \mathrm{C}(\mathrm{E}), 25^{\circ} \mathrm{C}(\mathrm{F})$, and $29^{\circ} \mathrm{C}(\mathrm{G})$. The y-axis represents the strength of Wolbachia life-shortening phenotype (estimated using Cox mixed models) and the $\mathrm{x}$-axis the Wolbachia doubling time (in days). The Pearson correlation coefficient $(r)$ and its significance ( $p$ ) are given in each panel.

https://doi.org/10.1371/journal.pgen.1009612.g005 
( $p<0.001$, for each comparison with $w$ MelCS_b). This effect is much stronger at $25^{\circ} \mathrm{C}$ than at $18^{\circ} \mathrm{C}(p<0.001$ for contrasts between both lines and $w$ MelCS_b $)$, and similar at $25^{\circ} \mathrm{C}$ and $29^{\circ} \mathrm{C}$ ( $p>0.21$ for these contrasts). At these two higher temperatures the lines carrying the variants with high copy number of Octomom have the shortest lifespan of all tested lines ( $p<0.001$ for all comparisons). $w$ MelPop2 and $w$ MelPop with low copy number of Octomom (2-3 copies) always have a weaker effect on host lifespan shortening than high copy number variants ( $p<0.001$ for all these comparisons). As observed with the high copy number variants, their effect increases with temperature, being stronger at $25^{\circ} \mathrm{C}$ than at $18^{\circ} \mathrm{C}$, and even stronger at $29^{\circ} \mathrm{C}$ ( $p<0.05$ for these comparisons). In fact, $w$ MelPop2 and $w$ MelPop with low copy number are only pathogenic at $25^{\circ} \mathrm{C}$ and $29^{\circ} \mathrm{C}$, not at $18^{\circ} \mathrm{C}$. These data confirm the association of Octomom region amplification with host lifespan shortening, and the increase in the severity of this phenotype with an increase in Octomom copy number, and an increase in temperature.

In some comparisons $w$ MelPop2 and $w$ MelPop differ significantly in their pathogenic phenotype (Fig 5D). This could indicate that there were differences in this phenotype between these two lines. Therefore, and as done above in the analysis of proliferation, we repeated this experiment comparing the lifespan phenotype in $w$ MelPop2 and $w$ MelPop lines with a tightly controlled Octomom copy number (S12 Fig). At $25^{\circ} \mathrm{C}$ lines both $w$ MelPop and $w$ MelPop2 with 3 copies of the Octomom region had a shorter lifespan than the line with $w$ MelCS_b $(p<0.001)$, and no difference between them $(p=0.29)$. These results show that $w$ MelPop2 and $w$ MelPop have the same phenotype.

To further demonstrate that the life shortening phenotypes were due to the new Wolbachia variants, and not to EMS-induced mutations in the host nuclear genome, we performed reciprocal crosses between flies carrying $w$ MelCS_b and flies carrying either $w$ MelOctoless or wMelPop2 (with 3 or 8-9 copies of Octomom) and followed the survival of their female progeny at $29^{\circ} \mathrm{C}$. The female progeny from reciprocal crosses should be identical in the nuclear genome but differ in the Wolbachia variant, which is maternally transmitted. The life-shortening phenotype segregated maternally, thus demonstrating that the Wolbachia variants carried by the lines are the cause of the phenotypes (S15 Fig). The relative strength of the life-shortening phenotype of the progeny of the reciprocal crosses matches the strength of the phenotypes in the maternal lines, observed in Figs 5 and S14. Moreover, all the tested lines that inherited $w$ MelCS_b had a similar lifespan ( $p>0.78$ for all comparisons), indicating, as expected, no contribution of the host genotype in this set of experiments.

The life shortening phenotype of $w$ MelPop has been associated with its over-proliferation and higher titres since its discovery [22]. We tested if these phenotypes were correlated by taking advantage of the data on titres, proliferation and lifespan shortening that we collected from this set of variants at different temperatures. We found a negative correlation between the strength of the life-shortening phenotype and Wolbachia doubling time, at all temperatures (Fig 5E-5G, $|\mathrm{r}|>0.86, \mathrm{p}<0.027$, for all correlations). However, we found no significant correlations between the strength of the life-shortening phenotype and Wolbachia titres in $0-1$ day-old adults (S16 Fig, $p>0.05$ for all correlations). These results show that over-proliferative variants shorten the host lifespan and the strength of this phenotype correlates with their proliferation rates.

\section{Wolbachia variants with deletion or amplification of the Octomom region provide stronger protection against $\mathrm{DCV}$}

Previous studies established a link between Wolbachia titres and the strength of anti-viral protection [16-20]. To test if $w$ MelOctoless and $w$ MelPop2 also provide a stronger protection 


\section{A}

Percentage of flies alive after DCV infection

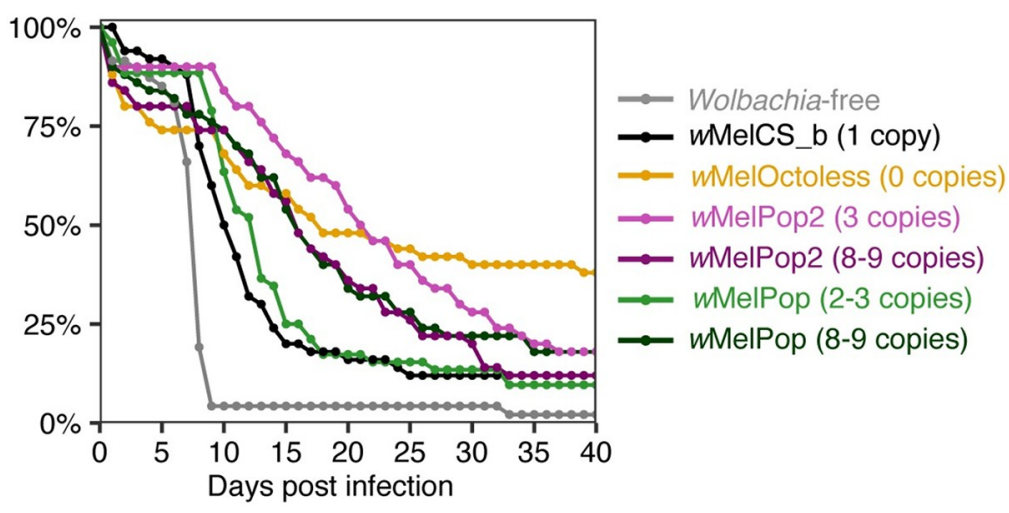

B

Coefficients Cox mixed model of DCV infected flies

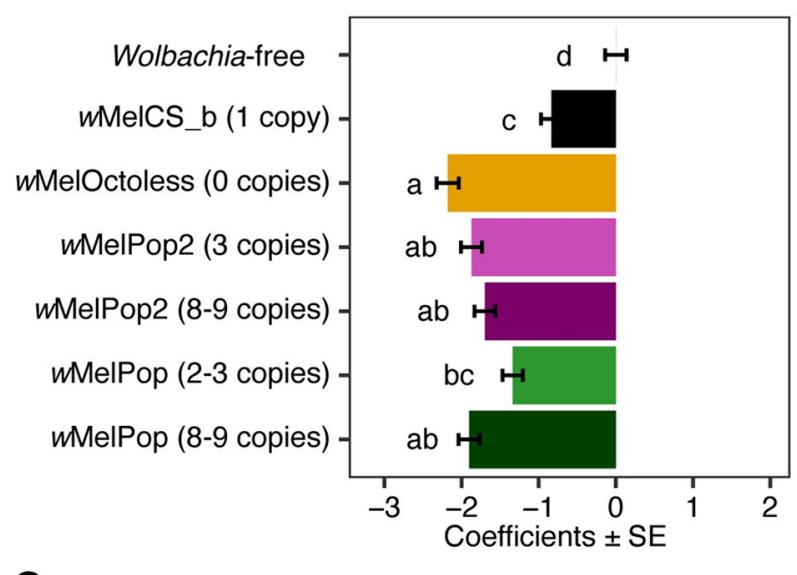

C

Anti-viral protection vs. Wolbachia titre at the day of eclosion

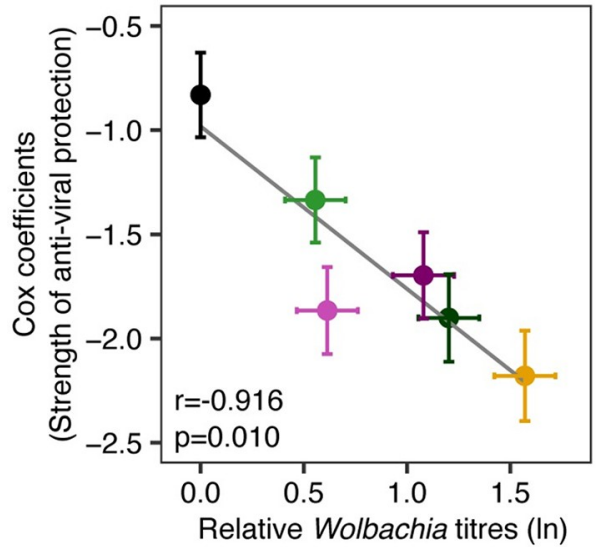

Fig 6. $w$ MelOctoless and $w$ MelPop2 provide strong protection against DCV. (A) Survival of males carrying different Wolbachia variants after a challenge with DCV. Fifty 3-5 day-old Drosophila males, per line, were pricked with DCV $\left(10^{9} \mathrm{TCID}_{50} / \mathrm{ml}\right)$ and survival followed for 40 days, at $18^{\circ} \mathrm{C}$. A replicate of the experiment and the bufferpricked controls are shown in S17 Fig. (B) Coefficients of Cox mixed models of DCV-infected flies. Cox coefficients 
represent the effect of Wolbachia infection on survival relative to the Wolbachia-free flies. Bars represent the standard error of the estimate, and the letters the statistically significant groups after $p$-value correction. Wolbachia infection improved the survival of DCV-infected flies $(p<0.001)$. (C) Correlation between the strength of anti-viral protection (represented as the coefficients of a Cox mixed model in the y-axis) and the natural log of Wolbachia titres at the day of eclosion, as a proxy for Wolbachia titre in the day of infection. The Pearson correlation coefficient $(r)$ and its significance $(p)$ are given in the panel.

https://doi.org/10.1371/journal.pgen.1009612.g006

against viruses, we infected flies with Drosophila C virus (DCV), by pricking, and followed their survival for 40 days at $18^{\circ} \mathrm{C}$. All Wolbachia variants tested provided protection against DCV (CHR, $p<0.001$ for all comparisons with the Wolbachia-free line, Figs 6A and 6B and S17A), while survival of Wolbachia-carrying flies did not differ from control when pricked with buffer, in this time frame (S17B-S17D Fig, $p=0.52$ for Wolbachia variant effect). $w$ MelCS_b was the least protective variant, while $w$ MelOctoless was the one providing the highest protection. In general, the over-proliferative Wolbachia variants confer stronger protection to DCV than $w$ MelCS_b, although this difference is not always significant (Fig 6B, $p<0.001$ for all comparisons, except for $w$ MelPop (2-3 copies), $p=0.11$ ).

We also tested the correlation between the antiviral protection and Wolbachia proliferation, but we found no correlation neither with proliferation estimates at $18^{\circ} \mathrm{C}(p=0.21)$, the temperature in which the flies were kept after infection, nor at $25^{\circ} \mathrm{C}(p=0.35)$, the temperature in which flies developed and were kept until being infected with DCV (S16D and S16E Fig). However, there is a significant correlation between the strength of Wolbachia-induced antiviral protection and Wolbachia titres in 0-1 day-old flies, a proxy for Wolbachia titre at the day of infection, which is 3-5 day-old flies (Fig 6C, $p=0.010,|r|=0.92$ ). Overall, the new over-proliferative variants give more protection to viruses than $w \mathrm{MelCS}$ _b, and the strength of this protection is correlated with Wolbachia levels at the time of infection.

\section{Discussion}

We developed a new forward genetic screen and identified new Wolbachia over-proliferative variants. We characterized in detail two of these mutants, $w$ MelPop2 and $w$ MelOctoless, and identified the genetic bases of their over-proliferation. $w$ MelPop2 had an amplification of the Octomom region, which has been previously shown to lead to over-proliferation in the $w$ MelPop variant $[16,17]$. $w$ MelOctoless, on the other hand, had a deletion of this same Octomom region. These results further confirm and develop the complex role of this genomic region in the control of Wolbachia proliferation. An extensive phenotypic characterization of two of these lines showed both Wolbachia variants to shorten the host lifespan, as well as to increase antiviral protection. Moreover, we show that Wolbachia proliferation rate in D. melanogaster depends on the interaction between Octomom copy number, the host developmental stage, and temperature. Our analysis also suggests that the Wolbachia-induced life shortening and antiviral protection phenotypes are dependent on its rate of proliferation in adults and titres near the time of infection, respectively. These are related, but different, properties of the endosymbiont.

\section{An unbiased approach for genetically intractable symbionts}

Given their dependence on the intracellular niche, Wolbachia and most endosymbionts remain non-culturable and genetically intractable, hindering their study. Here we aimed at mutagenizing and screening for new Wolbachia variants in the host. We expected that a main difficulty of this approach would be how to identify, via its phenotype, a new mutant present in the Wolbachia population within a host. We estimated here that a newly emerged 
wMelCS_b-carrying female harbours approximately 540,000 Wolbachia genome copies, which probably corresponds to the same number of Wolbachia cells [30]. Since EMS induces random mutations, we expected mosaicism in the Wolbachia population at the individual fly level. Each new mutant, when generated, would be a unique cell within these approximately half a million other Wolbachia cells. Since the Wolbachia phenotypes are normally measured at the individual host levels (e.g. Wolbachia titres, antiviral protection), the properties of individual or small numbers of mutant Wolbachia could be diluted and unmeasurable.

We hypothesized, however, that over-proliferating Wolbachia cells could overtake the population and that the resulting higher titres could be detectable. Indeed, fast proliferative Wolbachia can be selected at the level of a single host [31]. To increase the probability of isolating rare over-proliferating Wolbachia variants, we also relied on the bottleneck imposed in the vertical transmission of Wolbachia. We calculated here that single embryos carry approximately 3,000 Wolbachia genomes, which is consistent with previous estimates [29]. We also treated flies with tetracycline before mutagenesis to further reduce the Wolbachia population. We expected this additional endosymbiont titre reduction to enhance genetic drift and potentially enrich or lead to the fixation of rare Wolbachia variants. By screening at the immediate progeny (F1) of EMS-treated females or three generations later (F4) we were able to select new over-proliferative Wolbachia mutants.

\section{Genetic bases of Wolbachia over-proliferation}

After discarding the possibility that mutations in the host nuclear or mitochondrial genomes were the cause of Wolbachia over-proliferation, we performed de novo genome assembly of the ancestral, $w$ MelCS_b, and the new variants, $w$ MelPop2 and $w$ MelOctoless. We also assembled $w$ Mel and $w$ MelPop genomes. The assemblies generated complete full chromosomes of these Wolbachia and allowed us to identify single nucleotide differences and structural differences between these genomes. To validate our genome assembly pipeline we compared our $w$ Mel genome to the reference $w$ Mel genome. We identified only seven indels and two SNPs, which we confirmed to be present in our line, by Sanger sequencing. These indels and SNPs were also recently identified in a $w$ Mel that was transferred to D. simulans [32]. Our assembly results also showed two previously identified SNPs between $w$ MelCS_b (but also the new $w$ MelCS_b derived variants $w$ MelOctoless and $w$ MelPop2) and $w$ MelPop. Additionally, our assembly provides an improvement over the previous $w$ MelPop genome [25].

The only differences between the new over-proliferative variants and $w$ MelCS_b were structural differences in the Octomom region. wMelPop2 has an amplification of this region. The assembly confirms that the Octomom region is amplified in tandem [17], and that all copies are located in the Wolbachia genome. Previously we showed that amplification of the Octomom region and the degree of this amplification determined $w$ MelPop over-proliferative phenotype $[16,17]$. Moreover, reversion of $w$ MelPop Octomom copy number to one, through selection, resulted in loss of both over-proliferation and cost to the host, making the variant phenotypically identical to $w$ MelCS_b, which also carries a single copy of Octomom [17]. We now show that Octomom amplification in $w$ MelCS_b also leads to an over-proliferative phenotype. Moreover, $w$ MelPop and $w$ MelPop2 variants, carrying the same copy number of Octomom, have identical phenotypes. These findings are unsurprising since $w$ MelCS_b, the ancestor of $w$ MelPop2, and $w$ MelPop share an almost identical genome, differencing on two synonymous SNPs and Octomom amplification. Hence, our results further confirm the role of amplification of Octomom region in the over-proliferation of Wolbachia.

$w$ MelOctoless, on the other hand, has a deletion of the entire Octomom region. The deletion leaves behind one of the direct repeats flanking the Octomom region [16], suggesting that 
excision might have been mediated by recombination. The causal link between Octomom deletion and over-proliferation is further supported by an additional independent over-proliferative variant isolated in the screen, $w$ MelOctoless2. This Wolbachia variant also has a deletion of Octomom as the only difference with $w$ MelCS_b. These data show that deletion of the Octomom region also leads to an over-proliferative phenotype in Wolbachia. Thus, we identified the second known Wolbachia mutation with a clear link between genotype and phenotype.

The mutations identified in the new variants are deletions and amplifications. We did not detect any new SNPs in these variants, even if EMS is expected to mainly induce single nucleotide mutations [33]. Thus, it is possible that the deletion or amplification of the Octomom region in these over-proliferative variants were independent of the EMS treatment. For instance, loss of the Octomom region has been twice reported, in cell culture [25,34], suggesting that it may occur spontaneously. Yet, chemical mutagens such as EMS can activate DNA damage response and transposable elements [35], and some genes of the Octomom region and its flaking genes are predicted to be potentially involved in transposition and DNA repair [16]. Therefore, we cannot rule out that the EMS treatment induced the mutations in this genomic region.

\section{Opposing mutations lead to a similar Wolbachia over-proliferative phenotype}

Both the deletion or the amplification of the Octomom region causing an over-proliferative phenotype seems to be a paradox. The resolution of this paradox and the mechanisms leading to these phenotypes will rely on the functional characterization of genes in the Octomom region. These genes may be involved in interaction with the host, transcriptional regulation or DNA repair [16].

One possibility is that the amplification of the Octomom region and over-expression of a particular set of genes in this region lead, mechanistically, to the same result as the absence of the genes. There are many examples of over-expression of a gene leading to a dominant negative phenotype. For instance, both over-expression or loss of a protein forming a gradient, abolish the gradient [36]. Also, over-expression of a member of protein complex may lead to loss of stoichiometry and therefore loss of functional complexes [37]. Another possibility is that the loss and amplification of different genes in the Octomom region lead to the over-proliferative phenotype. The second hypothesis is supported by the fact that $w$ MelPop2 and $w$ MelOctoless have similar but not identical phenotypes, and interact differently with temperature. Furthermore, $w$ MelPop and $w$ MelPop2 have a higher rate of proliferation than $w$ MelOctoless at $25^{\circ} \mathrm{C}$ and $29^{\circ} \mathrm{C}$. Therefore, at these temperatures, the phenotype of Octomom amplification is stronger than the phenotype associated with the Octomom region loss-of-function.

Although complex, these results help to explain some of the data from an over-proliferative variant trans-infected in Aedes aegypti. The D. melanogaster variant $w$ MelPop was transinfected into Aedes albopictus cells, and then transinfected into A. aegypti. In the process of cell culture adaptation the Octomom region, which was amplified in $w$ MelPop, was deleted [25]. The $w$ MelPop-PGYP variant in A. aegypti lacks, therefore, the Octomom region. Nonetheless, this variant still over-proliferates and is highly pathogenic in A. aegypti [21]. If one assumed the same genetic basis for the pathogenicity of $w$ MelPop in D. melanogaster and $w$ MelPopPGYP in A. aegypti, one might conclude that the Octomom region was not related with these phenotypes [25]. However, similarly to wMelPop/wMelPop-PGYP variants our results with $w$ MelCS_b and the mutant variants show that both amplification and deletion of the Octomom region lead to increased Wolbachia pathogenicity. Thus, deletion of the Octomom region in $w$ MelPop-PGYP may explain why this variant is also pathogenic. However, $w \mathrm{Mel}-$ Pop-PGYP also accumulated other mutations when passaged in cell culture, and has many 
other genetic differences with $w \mathrm{Mel}$ [25]. These may also contribute to $w$ MelPop-PGYP being more pathogenic than $w \mathrm{Mel}$ in A. aegypti.

The Octomom region has the properties of a genomic island: it is not part of the Wolbachia core genome, since many Wolbachia strains lack this region, and seems to be horizontally transferred between Wolbachia strains $[16,38,39]$. Although the $w$ Mel strain can lose this region and remain viable in laboratory conditions (here and $[25,34]$ ), natural variants of $w \mathrm{Mel}$ without Octomom are not known [40]. Its over-proliferative phenotype, and the associated shortening of host lifespan, may lead to a fitness disadvantage to a host harbouring such mutant. This in turn may lead to loss of these variants from the host natural populations. Therefore, this genomic region, absent in many other strains of Wolbachia, became addictive to the $w$ Mel strain through its integration in the regulation of Wolbachia proliferation.

\section{Wolbachia proliferation, pathogenicity, and antiviral protection}

We found a complex interaction between temperature and proliferation of the different Wolbachia variants in adults. $w$ MelOctoless proliferates faster than $w$ MelCS_b, to a similar extent, at all temperatures. $w$ MelPop and $w$ MelPop2, however, strongly interact with temperature and proliferate much faster at higher temperatures. We also confirmed here that the degree of Octomom amplification in these variants modulates the proliferation rate [17], and interacts with temperature.

Throughout the range of tested temperatures, the different variants have very different proliferation rates in adults. At $25^{\circ} \mathrm{C}$, where we observed the highest variation, the titres of $w$ MelPop2 double every 2 days, while wMelCS_b titres double every 14 days. In contrast, during larval development the proliferation rates of $w$ MelCS_b, $w$ MelOctoless, and $w$ MelPop2, are very similar and much faster than in adults. During development, at $25^{\circ} \mathrm{C}$, Wolbachia titres double every 12 to 16 hours. These results show that $w$ MelCS_b, which has a relatively low proliferation rate in adult flies, is capable of very fast proliferation during larval development, when the host is rapidly growing. In Brugia malayi, Wolbachia titres also increase rapidly during the first few weeks of the nematode's development [30]. Thus, rapid proliferation during immature stages may be a conserved Wolbachia strategy to recover from the bottleneck imposed during maternal transmission. This observed coordination between $w$ MelCS_b proliferation and D. melanogaster developmental stage may be due to Wolbachia directly responding to host developmental cues, to differences in the metabolic profile of larvae and adults, or to differences in host cell divisions rates. Wolbachia could also control its proliferation in response to its own population density within the host.

While $w$ MelPop and $w$ MelPop2 proliferate similarly to $w$ MelCS_b during larval stages, they over-proliferate in adults. Thus, this over-proliferative phenotype of variants with amplification of the Octomom region can be interpreted as an inability to properly respond to the conditions of the host adult stage. On the other hand, the deletion of Octomom seems to lead to a similar increase in the proliferation rate during development and adult life, although the difference with $w$ MelCS_b is not significant during development in our analysis.

The new over-proliferative variants shorten the lifespan of D. melanogaster, as wMelPop does. Furthermore, we showed this phenotype to result from the interaction of Wolbachia genotype and temperature. $w$ MelOctoless had a similar life shortening phenotype at all temperatures, although stronger at $18^{\circ} \mathrm{C} . w \mathrm{MelPop}$ and $w$ MelPop2 responded strongly to temperature, being much more costly at higher temperatures, as shown before for $w$ MelPop $[17,22,41]$. The extent of Octomom amplification also influenced this interaction. While low copy number variants had no phenotype at $18^{\circ} \mathrm{C}$, the high copy number $w$ MelPop and $w$ MelPop2 are pathogenic also at this temperature. Therefore, these variants can also be pathogenic at $18^{\circ} \mathrm{C}$, contrary to previous data $[28,41,42]$. Interestingly, we find at all temperatures a 
significant correlation between the proliferation rate of the Wolbachia variants and the life shortening phenotype. The faster the variants proliferate the shorter the host lifespan.

All the over-proliferative variants also increased antiviral resistance, with $w$ MelOctoless conferring the strongest protection. This phenotype correlated poorly with proliferation rates at $25^{\circ} \mathrm{C}$ or $18^{\circ} \mathrm{C}$, the temperature before and after infection with DCV, respectively. However, the strength of the antiviral resistance correlated with the titres of Wolbachia near the day of infection. Thus, the cost of harbouring Wolbachia in terms of lifespan, and the benefit of the antiviral protection, correlate with related but different parameters. In the future it will be important to understand why these different correlations. For instance, Wolbachia titres at the point of viral infection are probably important because the anti-viral protection is observed early in the infection [43]. Wolbachia proliferation rate could impact longevity either by the cumulative cost of the proliferation process itself or by determining the time to reach a lethal threshold of Wolbachia titres. Nonetheless, these results indicate that it may be possible to select for highly protective Wolbachia variants without necessarily having a high cost to the host. These would be Wolbachia variants with high titres but low proliferation in adults. Such variants would be particularly useful in the use of Wolbachia-transinfected mosquito to prevent arboviruses transmission.

In summary, our results show the feasibility of forward genetic screens to study Wolbachia biology. Similar strategies may be used in the future to study other aspects of Wolbachia-host interactions or the biology of other genetically intractable endosymbionts. The new over-proliferative variant $w$ MelPop2 confirms the causal link between amplification of the Octomom region and Wolbachia over-proliferation. Whereas the new loss-of-function mutant $w$ MelOctoless reveals that this region is also required to control Wolbachia proliferation. These results give new insight on the complex role this genomic region plays in Wolbachia biology. Moreover, this collection of variants, similarly to an allelic series, allow a finer dissection of the consequences of Wolbachia over-proliferation to the host.

\section{Materials and methods}

\section{Fly genotypes, infection status, and maintenance}

Flies were reared on fly food, supplemented with live yeast, at $25^{\circ} \mathrm{C}, 70 \%$ humidity. Fly food was composed of molasses $(37.5 \mathrm{~g} / \mathrm{L})$, sugar $(62.5 \mathrm{~g} / \mathrm{L})$, cornflour $(58.3 \mathrm{~g} / \mathrm{L})$, yeast extract $(16.7 \mathrm{~g} / \mathrm{L})$, and agar $(8.3 \mathrm{~g} / \mathrm{L})$ in distilled water. The mixture was sterilized by autoclaving and cooled to $45^{\circ} \mathrm{C}$. For each litre of food, we added $29.2 \mathrm{~mL}$ of a solution with $100 \mathrm{~g}$ of methylparaben and $0.2 \mathrm{~g}$ of Carbendazim for $1 \mathrm{~L}$ absolute ethanol.

All fly stocks used had the Drosdel $w^{1118}$ isogenic background [16,44].

The bacterial community associated with the fly stocks was homogenized as in Pais et al. [45], with minor modifications. Briefly, we collected eggs for 6 hours in fresh agar plates with live yeast and sterilized the eggs surface by consecutive washes on $2.1 \%$ sodium hypochlorite $(\mathrm{NaOCl})$ solution (10 minutes), 70\% ethanol (5 minutes) and sterile water (5 minutes). Next, we transferred axenic eggs to sterile fly food supplemented with $40 \mu \mathrm{L}$ of 1:1 overnight culture of Acectobater OTU 2753 and Lactobacillus OTU 1865 [45]. We confirmed the presence of these bacterial species by squashing five females aged 3-6 days in sterile $1 \times$ PBS, plating $30 \mu \mathrm{L}$ of the lysate in mannitol plates, incubate them at $25^{\circ} \mathrm{C}$ for $72 \mathrm{~h}$, and identify bacteria by colony morphology.

\section{Selection of D. melanogaster lines carrying Wolbachia with specific Octomom copy number}

To select for flies carrying $w$ MelPop and $w$ MelPop 2 with a desired Octomom copy number, we proceeded as in Chrostek and Teixeira [17], with minor modifications. Briefly, we allowed 
5-20 virgin females to cross with 2-3 Wolbachia-uninfected males of the Drosdel $w^{1118}$ isogenic background in individual vials, and lay eggs for 3-4 days. Females were then collected in individual tubes for DNA extraction and Octomom copy number determination by qPCR. The progeny of females with specific copy numbers were then followed-up.

\section{Determination of time for Wolbachia titres recovery}

Flies with $w$ MelCS_b developed in fly food supplemented with tetracycline at the concentrations $1.5625 \mu \mathrm{g} / \mathrm{ml}, 3.125 \mu \mathrm{g} / \mathrm{ml}, 6.25 \mu \mathrm{g} / \mathrm{ml}, 12.5 \mu \mathrm{g} / \mathrm{ml}, 25 \mu \mathrm{g} / \mathrm{ml}$, and $50 \mu \mathrm{g} / \mathrm{ml}$. Three isofemale lines were established from each dose. In the F1, we randomly selected four virgin females for egg-laying and Wolbachia titre measurement using qPCR. From this moment on, the flies were kept on fly food without tetracycline. We set up the next four generations using the progeny of a female with the median Wolbachia titres.

\section{Forward genetic screen}

We attempted to mutagenize Wolbachia in vivo by feeding its host with the mutagen EMS. DrosDel $w^{1118}$ isogenic flies carrying $w$ MelCS_b were raised in standard fly food or fly food supplemented with tetracycline (from 1.5625 to $12.5 \mu \mathrm{g} / \mathrm{ml}$ ). Virgin females were collected, starved for $6 \mathrm{~h}$, and then fed EMS concentrations ranging from 10 to $8,000 \mathrm{mM}$ diluted in $1 \%$ sucrose. Control flies fed on sucrose solution only. A dye was added to the feeding solution to confirm intake and feeding proceeded for $13 \mathrm{~h}$ (overnight).

EMS-fed females (G0), and control females, were mated individually with 2-3 Wolbachiafree Drosdel $w^{1118}$ isogenic males, egg-laying was allowed for 3-4 days, and parents discarded. From the F1 progeny, we collected virgin females, mated them individually with 2-3 Wolbachia-free Drosdel $w^{1118}$ isogenic males, and allowed egg laying for 3-4 days. These females were collected when 10 day-old, and Wolbachia titres determined by qPCR. We followed the progeny of F1 females showing 50\% or higher increase in Wolbachia titres relative to control flies in the same conditions. We also transferred the progeny of these F1 for three more generations, without selection, and repeated the determination of Wolbachia titres in F4 females. In the same experimental batch we may have tested more than one F1 or F4 progeny from each G0 female. Hence, over-proliferative Wolbachia variants isolated in the same batch of treated females may be a result of a single event in the G0 female.

\section{Real time quantitative PCR}

DNA extraction for qPCR was performed as described before [17].

The qPCR reactions were performed in the QuantStudio 7 Flex (Applied Biosystems). The reaction mix and PCR program used were described before [16]. The specificity of the amplicons was confirmed by analysing the melting curve profile.

Relative levels of the target genes were determined using the Pfaffl method [46]. To quantify relative Wolbachia titres we used Drosophila RpL32 gene as calibrator, and Wolbachia wsp as the target gene. To determine the copy number of the Octomom region, Wolbachia wsp gene was used as the calibrator and WD0513 used as the target gene. For determination of copy number of other Octomom region genes, or control genes, $w s p$ was also used as a calibrator.

For absolute quantification of Wolbachia genome copies the full-length Wolbachia wsp gene was cloned into a pMT/V5 Drosophila expression vector (Invitrogen). The plasmid was amplified in Escherichia coli strain DH5- $\alpha$, purified using midiprep (QIAGEN) and its concentration determined using Qubit 2.0 (Thermo Fisher Scientific). Molecular weight of the plasmid was calculated assuming a nucleotide average weight of $325 \mathrm{Da}$ to determine the number 
of plasmid molecules in the calibration curve. Standard curves of 1:10 serial dilutions were run to calibrate the assay each time.

Primers used in $\mathrm{qPCR}$ reactions are given in S8 Table.

\section{Determination of Drosophila lifespan}

For each replicate, a total of 50 males or 50 females were collected on the day of eclosion. Flies (10 per vials) were then incubated at $18^{\circ} \mathrm{C}, 25^{\circ} \mathrm{C}$ or $29^{\circ} \mathrm{C}$, and transferred to new fresh vials every four (females) or five (males) days. The number of dead flies was recorded daily until all the flies died. Censored observations (i.e. flies lost or trapped in the vial plug) were recorded and taken into account during data analysis.

\section{Protection against Drosophila C Virus}

We produced and titrated the Drosophila C virus solution as described in Teixeira et al. [8]. We infected 50 3-5 day-old males by dipping insect needles (Austerlitz Insect Pins) into a virus solution $\left(10^{9} \mathrm{TCID}_{50} / \mathrm{ml}\right.$ in $50 \mathrm{mM}$ Tris- $\left.\mathrm{HCl}, \mathrm{pH} 7.5\right)$ and pricking in the thorax flies anaesthetized under $\mathrm{CO}_{2}$. An equal number of males were pricked with a buffer only solution (50mM Tris- $\mathrm{HCl}, \mathrm{pH} 7.5)$ and served as controls. After pricking, flies were incubated in groups of 10 individuals per vial, and kept at $18^{\circ} \mathrm{C}$. Survival was followed as above.

\section{Wolbachia proliferation during development and in adults}

To determine Wolbachia growth during development, flies carrying $w$ MelCS_b, wMelOctoless, and wMelPop2 (high-copy), laid eggs for 2 hours in apple juice agar plates supplemented with live yeast. Eggs were transferred to fly food-containing bottles and allowed to develop at $25^{\circ} \mathrm{C}$. For Wolbachia titre assessment, we sampled eggs (2 hours), L1 larvae (24 hours later), newly moulted L2 larvae (48 hours later), L3 larvae (72 and 84 hours), white prepupae pupae (120 hours), P8 staged pupae (168 hours), and newly eclosed adult males and females (240 hours). Ten samples per time point were analysed. Samples included ten individuals each for eggs and larvae and one individual each for pupae and adults. To collect newly molted larva, all larvae of the target stage were discarded at the respective time-point and the newly molted larvae were collected within two hours after the established time-point. The white prepupae were collected by staging and the remaining stages were collected according to the set timepoint. Except for adults, which were collected within 24 hours post-emergence, all samples were collected within two hours interval.

For assessment of titres dynamics in adult flies, newly eclosed males, raised at $25^{\circ} \mathrm{C}$, were incubated at $18^{\circ} \mathrm{C}, 25^{\circ} \mathrm{C}$, and $29^{\circ} \mathrm{C}$. Flies were collected every three $\left(29^{\circ} \mathrm{C}\right)$, seven $\left(25^{\circ} \mathrm{C}\right)$ or ten days $\left(18^{\circ} \mathrm{C}\right)$ for Wolbachia titre measurement. Ten individuals were processed for each time point, and the experiment was performed twice. Each sample consisted of a single fly.

\section{Wolbachia genomes sequencing and quality control}

For Wolbachia genomic sequencing (Illumina and Oxford Nanopore), we enriched the sample for Wolbachia cells before DNA purification. To this end, approximately 50010 day-old flies were squashed for 5 minutes in 10ml Schneider's Insect Medium (Thermo Fisher Scientific) using glass beads. Next, we pelleted host debris by centrifugation at 1,000g for $5 \mathrm{~min}$ and filtered the supernatant solution thought a $5 \mu \mathrm{m}$ pore. Bacterial cells were pelleted by centrifugation at 13,000rpm for 15 minutes, and DNA was extracted. All centrifugations were carried out at $4{ }^{\circ} \mathrm{C}$. DNA was extracted with a phenol-chloroform isolation protocol and resuspended in $10 \mathrm{mM}$ Tris- $\mathrm{HCl}(\mathrm{pH} 8)$. 
Sequencing was performed at the Genomics Facility at the Instituto Gulbenkian de Ciência, Portugal. Both Illumina and Oxford Nanopore sequencing was done on genomic DNA extracted from the same biological material. Illumina $300 \mathrm{bp}$ paired-end libraries were prepared using the Pico Nextera kit according to the manufacturer's instructions and sequenced with MiSeq. Data quality was assessed via FastQC v.0.11.5 [47] and reads were trimmed using Trimmomatic v.0.36 [48]. Genomic samples for Oxford Nanopore sequencing were processed with minimal shearing to maximize the size of fragments in the libraries. After ligation (kit SQK-LSK108), libraries were sequenced in MinION Mk1b portable sequencing device using SpotON flow cell (R9.4.1). The status of the sequencing pores was monitored using MinKNOW (v2.0.1). Sequencing lasted for up to 48 hours. Albacore (v2.3.1) and Porechop (v0.2.2) were used for base-calling and read trimming, respectively.

\section{Genome assemblies and comparison}

Illumina and Oxford Nanopore reads were first mapped to D. melanogaster genome (BioProject: PRJNA13812) using BWA mem v0.7.12-r1039 [49] and minimap2 v2.17-r941 [50], respectively. Reads mapping to $D$. melanogaster genome were removed from the datasets before proceeding with Wolbachia genome assembly. We used Unicycler v0.4.8-beta [51] assembly pipeline on the remaining reads in order to assemble the Wolbachia genomes. Briefly, Unicycler uses Illumina reads to produce a repeats-limited image graph using Spades v3.9.0 [52], which was further refined through Bandage v0.8.1 [53]. Both small short nodes and nodes with no homology with $w$ Mel genome (AE017196.1) upon blastn v2.8.1+ [54] search were removed. Next, repeats were resolved by bridging Spades assemblies with Oxford Nanopore long reads. The resulting draft assemblies were polished using Racon v1.3.1 [55] and Pilon v1.23 [56] and rotated so that genomes begin at the dnaA gene (draft 1 genomes, not published).

We further refined our genome assemblies by mapping the Illumina reads to the corresponded draft genomes to identify mismatches, which were later corrected via Sanger sequencing (draft 2 genomes, not published). Primers used are in S9 Table.

Next, we compared the draft 2 genome assemblies by aligning $w$ MelPop, $w$ MelCS_b, $w$ MelPop2, and $w$ MelOctoless using Mauve v2.4.0 [57]. The differences between these genomes could correspond to differences between Wolbachia variants or still genome assembly artefacts. All detected differences were analysed by Sanger sequencing (primers in S9 Table). There were no confirmed SNPs or small indels between $w$ MelCS_b, $w$ MelPop2, and $w$ MelOctoless. However, we identified and confirmed using Sanger sequencing two predicted SNPs between wMelPop and the other Wolbachia variants. The genomes were corrected with the Sanger sequencing information and published (BioProject: PRJNA587443).

We further tried to identify mutations in the over-proliferative variants following a previously published pipeline [58]. It consisted of mapping quality checked reads to a reference genome using BWA mem v0.7.12-r1039 algorithm [49] and saving the output as Sequence Alignment/Map file format (SAM). After conversion to the Binary Alignment/Map format (BAM), the file was sorted, duplicates removed and indexed using SAMtools v0.1.19 [59]. Next, we generate mpileup files, also using SAMtools (option '-d 1,000,000'), after which was converted to Variant Call Format (VCF) files using BCFtools v1.9-209. We visually confirmed all inferred mutations in IGV v2.4.2 [60]. We did not consider mutations associated with homopolymer regions or in regions with low coverage $(<10 \mathrm{X})$. The set of mutations were compared between Wolbachia variants using custom Python and R scripts. The only difference we detected between these genomes was higher coverage or deletion of Octomom region.

To compare the set of mutations in the mitochondria of flies infected with different Wolbachia variant, we mapped Illumina reads to the D. melanogaster Release 6 genome sequence 
(KJ947872.2:1-14,000) and proceed as previously. Mutations following the criteria previously described were also compared by using custom scripts.

\section{Statistical analysis}

All the statistical comparisons were performed in R v4.0.0 [61].

To compare Wolbachia titres across multiple groups, we used linear models (LM) or linear mixed models (LMM). The effect of EMS on Wolbachia titre was tested using non-linear regression. We estimated the doubling time of Wolbachia variants using the equation $\log (2) / \beta$, with $\beta$ being the coefficients of an exponential model.

The lifespan datasets and survival curves after challenge with DCV were analysed with mixed effect Cox models [62].

The significance of correlations were tested using Pearson's correlation coefficient.

If multiple comparisons were necessary, the p-values were adjusted as proposed by Holm [63]. When multivariate techniques were applied, all the relevant covariates were included in the model, and the final model was selected as proposed by Burnham \& Anderson [64].

All statistical analysis and supporting data is deposited in https://doi.org/10.6084/m9. figshare.14079920.v1 [65].

\section{Supporting information}

S1 Fig. Wolbachia recovers from severe titre reduction within four fly generations. Relative Wolbachia titres of the progeny of tetracycline-treated flies. wMelCS_b-carrying females laid eggs in food containing varying doses of tetracycline. The progeny of three females were used to set up the experiment. At the first generation, four females were randomly selected for egglaying in antibiotic-free fly food and Wolbachia titre was measured using qPCR. Titres of untreated females were used to normalize the qPCR results. The progeny of a female with the median titre was used to set up the next generation. Wolbachia titre in the F1 was significantly determined by the concentration of the antibiotic $(p<0.001$ for all doses compared with control at generation 1), but recovered to normal within four fly generations ( $p>0.05$ for all doses compared with control at generation 4).

(TIFF)

S2 Fig. EMS decreases female fecundity and Wolbachia titre in the next generation in a dose-dependent manner. The total number of eggs (A) and adults (B) from females treated with varying doses of EMS. The reproductive output of 10 females was determined in the first ten days after EMS treatment by daily transferring females to new vials for egg laying. Females fed on a sucrose solution served as controls. Each dot represents the total number of eggs (A) or adults (B) laid by individual females during ten days. The effect of EMS on the reproductive output of females was estimated using a non-linear model and was highly significant ( $p<0.001$ for both numbers of eggs and adults per female). (C and D) Wolbachia titres in the F1 progeny of females treated with varying EMS doses. Wolbachia titre was quantified on individual females ( $\mathrm{n}=5-13$ per dose), after laying eggs for three days. Wolbachia titres were normalized against the titres of untreated females. Dashed red lines represent the mean value predicted using non-linear models. The effect of EMS on Wolbachia titres in the next generation was highly significant ( $p<0.001$ for both panels).

(TIFF)

S3 Fig. Isolation of over-proliferative Wolbachia variants. (A-D) Relative Wolbachia titres in a control (wMelCS_b) and EMS-treated D. melanogaster lines. Flies to set up the next generation was selected as described for Fig 1. Line 2B was isolated in the same batch as Line 2A 
(wMelOctoless) and they may be not independent. Likewise, Lines 3A (wMelOctoless2), 3B, and $3 \mathrm{C}$ were also isolated in a same batch.

(TIFF)

S4 Fig. Generation of isogenic D. melanogaster lines with $w$ MelPop2 and $w$ MelOctoless. The first, second and third chromosomes of flies carrying $w$ MelPop2, $w$ MelOctoless, and $w$ MelOctoless 2 were replaced through the use of balancer chromosomes. Wolbachia infection (and also mitochondria) was kept in the stock by crossing females with Wolbachia with indicated males. The mitochondria are only shown in females because of its strictly maternal transmission. All males were free of Wolbachia infection. Dashed lines indicate the genotype selected from the previous cross. Virgin female in the first cross were considered mutant in all chromosomes $\left(^{*}\right)$, for illustrative purposes. Question marks (?) represent recombined chromosomes.

(TIFF)

S5 Fig. Proliferation of $w$ MelOctoless and $w$ MelOctoless 2 in a host isogenic genetic background. Relative Wolbachia titres in D. malanogaster males carrying $w$ MelOctoless and $w \mathrm{Me}$ lOctoless 2 at 0 and 7 days post adult eclosion, at $25^{\circ} \mathrm{C}$. This experiment was set-up as described in Fig 1. Relative Wolbachia titre was determined using qPCR and normalized to that of $0-1$ days-old $w$ MelCS_b-infected males. Each dot represents the relative titre of a single male.

(TIFF)

S6 Fig. Confirmation of amplification and deletion of Octomom genes by qPCR. The amplification and deletion of individual Octomom genes ( $w$ Mel loci WD0507-WD0514) was confirmed using qPCR in $w$ MelPop 2 and $w$ MelOctoless, respectively. The copy number of three genes outside the Octomom region ( $w$ Mel loci WD0505, WD0519, and rpoD) were also determined. Five females carrying $w$ MelCS_b, $w$ MelPop2, and $w$ MelOctoless were used in the analysis. The copy number of $w$ MelPop 2 and $w$ MelOctoless genes is relative to that of $w$ MelCS_b.

(TIFF)

S7 Fig. Selection for lines carrying Wolbachia with a desired Octomom copy number. The relative copy number of genomic WD0513 in Wolbachia-carrying stocks throughout 30 fly generations. Each generation, 5-20 females were randomly collected for egg-laying for 3-4 days and used to determine the relative copy number of WD0513, as a proxy for the Octomom copy number. The progeny of a single female was used to set up the next generation. qPCR results were normalized to that of $w$ MelCS_b, which has a single copy of Octomom per genome.

(TIFF)

S8 Fig. Octomom region is amplified in tandem in $w$ MelPop 2 and $w$ MelPop. Oxford Nanopore MinION reads supporting the amplification of the Octomom region in tandem in $w \mathrm{Mel}-$ Pop2 and $w$ MelPop Wolbachia variants. We mapped $w$ MelPop2 and $w$ MelPop long reads (BioProject: PRJNA587443) to the the Octomom region in their genomes (Accessions CP046922.1 and CP046921.1, respectively) using minimap2 v2.17-r941 [48] and plotted the alignment summary (S7 Table) for illustrative purposes.

S9 Fig. Identification of the genetic bases for over-proliferation of the Wolbachia in Line 2B and Line 3A (wMelOctoless2). Relative coverage in the genomic region containing the Octomom region. As in Fig 2B, Illumina paired-end reads were mapped to wMelCS_b 
(GenBank: CP046924.1) genome, and the number of reads mapping to each position were normalized by dividing to the median coverage across the genome. Coverage information for $w$ MelCS_b, $w$ MelPop2 and $w$ MelOctoless is also given in Fig 2B. We identified the deletion of Octomom as the cause of proliferation in lines $2 \mathrm{~B}$ and line $3 \mathrm{~A}$ ( $w$ MelOctoless2), as no other difference was found when compared to $w$ MelCS_b.

(TIFF)

S10 Fig. The amplification or deletion of Octomom increase Wolbachia proliferation rate in adults. Time-course of relative Wolbachia titres in adults at $18^{\circ} \mathrm{C}(\mathrm{A}), 25^{\circ} \mathrm{C}(\mathrm{B})$ and $29^{\circ} \mathrm{C}$ (C) with different Wolbachia variants. Replicate of experiment shown in Fig 3. Wolbachia titres were determined and analysed as described for Fig 3. (TIFF)

S11 Fig. Octomom copy number determines Wolbachia titres on the day of adult eclosion. Relative Wolbachia titres on the day of adults eclosion. Males developed at $25^{\circ} \mathrm{C}$ were collected within 24 hours after eclosion for Wolbachia titre measurement using qPCR. Data used in this figure are also shown in Fig 3 and S10 Fig (time point 0). Letters represent significant groups after $p$-value correction.

S12 Fig. $w$ MelPop2 and $w$ MelPop are phenotypically indistinguishable. (A) WD0513 copy number of $w$ MelPop2 and $w$ MelPop in two experimental replicates. Using WD0513 as a proxy, the Octomom copy number of $w$ MelPop 2 and $w$ MelPop was tightly controlled prior to phenotypic comparison. (B) Wolbachia relative titres at $25^{\circ} \mathrm{C}$. The progeny of $w \mathrm{MelPop} 2-$ and $w$ MelPop-infected females carrying three copies of Octomom was used to set up the experiments. Males that developed at $25^{\circ} \mathrm{C}$ were collected upon eclosion, aged to specific time-points and used to determine Wolbachia titres using qPCR. Wolbachia titres were normalized to that of $w$ MelCS_b-carrying flies collected on the day of eclosion. Proliferation rates of $w$ MelPop2 and $w$ MelPop were not different $(p=0.32$ ). (C) Lifespan of males (solid lines) and females (dashed lines) flies at $25^{\circ} \mathrm{C}$. Males were transferred to new vials every five days, while females every four days. (D) Coefficients of a Cox mixed model, representing the effect of $w$ MelPop2 and $w$ MelPop on the lifespan relative to $w$ MelCS_b-carrying flies. $w$ MelPop2 and $w$ MelPop was equally pathogenic $(\mathrm{p}=0.29)$.

S13 Fig. Octomom copy number dynamics throughout fly development and during adult life. Relative copies of WD0513 throughout D. melanogaster development (A) and during adult life (B). WD0513 relative copy numbers were determined in samples shown in Fig 4 (for panel A) and Fig 3 and S10 Fig (for panel B). WD0513 copies were normalized to that of 0-1 old $w$ MelCS_b-infected males. (A) Vertical dashed lines separate developmental stages (i.e. eggs, larvae, pupae, and adults). The $\mathrm{x}$-axis is not in a continuous scale. (B) The two replicates are represented by different symbols.

(TIFF)

S14 Fig. $w$ MelPop2 and $w$ MelOctoless are pathogenic to both males and females. Lifespan of D. melanogaster males at $18^{\circ} \mathrm{C}(\mathrm{A}), 25^{\circ} \mathrm{C}(\mathrm{B})$, and $29^{\circ} \mathrm{C}(\mathrm{C})$. Survivorship was determined as in Fig 5. This is a replicate of Fig 5. (D) Survival of D. melanogaster females at $25^{\circ} \mathrm{C}$. Survival was determined as in Fig 5, except that females were transferred to new vials every four days. The experiment was performed twice. (E) Coefficients of a Cox mixed model of the lifespan of females relative to Wolbachia-free control. Both replicate experiments were pooled for statistical comparisons. Bars represent the standard error of the coefficient, and letters the statistically 
significant groups.

(TIFF)

S15 Fig. Wolbachia variants, not differences in the host genetic background, are pathogenic. (A-B) Survival of D. melanogaster females at $29^{\circ} \mathrm{C}$. Virgin $w$ MelCS_b-carrying females were crossed with males carrying $w$ MelOctoless or $w$ MelPop2 (with 3 or 8-9 Octomom copies) and vice-versa. The resulting progeny developed at $25^{\circ} \mathrm{C}$ and was placed at $29^{\circ} \mathrm{C}$ after adult eclosion. The survival of 50 female progeny, which have the same genetic background but differ in Wolbachia infection, was determined per condition, per replicate. Females were maintained in groups of ten and transferred to new vial every four days. The experiment was performed twice. (C) Coefficients of a Cox mixed model representing the effect of the parental crosses on the survivorship of females. Significance was accessed after p-value correction for multiple comparisons, and significant groups are represented by letters.

(TIFF)

S16 Fig. Correlation between Wolbachia-induced phenotypes and bacterial titres or doubling time. (A-C) Correlation between Wolbachia titre at the day of eclosion and the strength of life-shortening phenotype determined at $18^{\circ} \mathrm{C}(\mathrm{A}), 25^{\circ} \mathrm{C}(\mathrm{B})$, and $29^{\circ} \mathrm{C}(\mathrm{C})$. The y-axis represents the strength of Wolbachia life-shortening phenotype (estimated using Cox mixed model shown in Fig 5). The $\mathrm{x}$-axis represents the natural log of the relative Wolbachia titre estimated using a linear mixed model. Bacterial titres were normalized to that of $w$ MelCS_binfected flies (shown in S11 Fig). (D and E) The correlation between the strength of anti-viral protection and Wolbachia doubling time. The y-axis represents the strength of anti-viral protection (estimated using Cox mixed model shown in Fig 6). The x-axis represents Wolbachia doubling time in adults at $18^{\circ} \mathrm{C}(\mathrm{D})$, or $25^{\circ} \mathrm{C}(\mathrm{E})$ (shown in Table 1). The Pearson correlation coefficient (r) and its significance (p) are given in each panel. A grey line represents the trend (fit of linear regression). Error bars represent the standard errors of the estimates. None of these correlations were statistically significant and they complement correlations shown in Fig 5 and Fig 6.

S17 Fig. Survival of flies with different Wolbachia variants after challenge with DCV or buffer solution. (A) Survival of males carrying different Wolbachia variants after a challenge with DCV (A) or a buffer solution (B and C). Fifty 3-5 days-old Drosophila males, per line, were pricked with $\mathrm{DCV}\left(10^{9} \mathrm{TCID}_{50} / \mathrm{ml}\right)$ or buffer and survival curves were determined at $18^{\circ} \mathrm{C}$ for 40 days. $\mathrm{A}$ is a replicate of Fig $6 \mathrm{~A}, 6 \mathrm{~B}$ and $6 \mathrm{C}$ are controls for these experiments. (D) Coefficients of Cox mixed models of buffer-pricked flies. Both replicates were pooled for statistical analysis. Bars represent the standard error of the estimate, and the letters the statistically significant groups after p-value correction.

(TIFF)

S1 Table. Number of F1 females screened for new over-proliferative Wolbachia variants per experimental condition. $w$ MelCS_b-infected G0 females, raised in control or antibiotictreated food $(12.5 \mu \mathrm{g} / \mathrm{ml})$, were fed different doses of ethyl-methanesulfonate (EMS) and allowed to lay eggs in individual vials. F1 females were collected as virgins, mated to nonmutagenized males and also allowed to lay eggs individually. F1 females were used for Wolbachia titre measurement when were 10-days old. Number of F1 females tested per experimental condition is shown.

(XLS) 
S2 Table. Coverage statistics of the sequencing project. Coverage statistics (mean and range) of Illumina reads mapped to either Wolbachia or mitochondria of D. melanogaster Release 6 genome sequence (KJ947872.2:1-14,000). Sequencing data of each Wolbachia variants are mapped to own genome assembly (BioProject ID: PRJNA587443), except for Wolbachia in Line $2 \mathrm{~B}$ and $w$ MelOctoless 2 which were mapped to $w$ MelCS_b genome (Accession: CP046924.1). ND-not determined. (XLS)

S3 Table. Flies infected with new over-proliferative Wolbachia variants did not inherit mutated mitochondria. Illumina reads on flies infected with different Wolbachia variants were mapped to the mitochondria of $D$. melanogaster Release 6 genome sequence (KJ947872.2:1-14,000). A summary of the mapping is given in S2 Table. The mitogenome of flies infected with $w$ MelCS_b, wMelOctoless and $w$ MelPop2 was identical. We found an SNP unique to flies infected with $w$ MelCS-like variants $(\mathrm{G} \rightarrow \mathrm{A}$ on position 10,793$)$ but absent in flies infected with $w$ MelPop. We confirmed this SNP using Sanger sequencing.

S4 Table. Assembly and annotation statistics. Wolbachia genomes were assembled using the Unicycler v0.4.8-beta pipeline and annotated using NCBI Prokaryotic Genome Annotation Pipeline v4.10. $w \mathrm{Mel}$ reference genome (Accession: AE017196.1) is included for comparison purposes.

(XLS)

S5 Table. SNPs and indels between newly assembled $w \mathrm{Mel}$ and $w \mathrm{Mel}$ reference genome. The genome of a newly assembled Cluster III wMel Wolbachia variant (Accession: CP046925.1) was aligned to $w$ Mel reference genome (Accession: AE017196.1) using Mauve v2.4.0. All the differences were confirmed via Sanger sequencing.

S6 Table. SNPs and indels between $w$ MelCS_b and and $w \mathrm{Mel}$ reference genome. The genome of $w$ MelCS_b (Accession: CP046924.1) was aligned to $w$ Mel reference genome (Accession: AE017196.1) using Mauve v2.4.0.

S7 Table. Alignment summary of long reads supporting the amplification of the Octomom region in tandem. Long reads (MinION, Oxford Nanopore) reads supporting the amplification of the Octomom region in tandem in wMelPop2 (Accession: CP046922.1) and wMelPop (Accession: CP046921.1) genomes. Long reads were mapped to Octomom region using minimap2 v2.17-r941 and the number of Octomom copies determined using blastn v2.8.1+. (XLS)

S8 Table. Primers used for amplification and quantification of individual Wolbachia genes. Primers used in this study have been previously described [16,17]. (XLS)

S9 Table. List of primers used to improve Wolbachia draft genomes. Primers used to amplify and sequence, using Sanger technology, genomic regions containing predicted differences between Wolbachia draft genomes.

S1 Text. Confirmation of the amplification and deletion of the Octomom in wMelPop2 and wMelOctoless, respectively. The genomes of $w$ MelCS_b, $w$ MelPop 2 and $w$ MelOctoless were aligned using Mauve v2.4.0. The three-fold amplification of Octomom in wMelPop2 and 
its deletion in $w$ MelOctoless were the only difference identified when compared with $w$ MelCS_b.

(TXT)

\section{Acknowledgments}

We are thankful to the Bioinformatics Unit and the Genomics Facility at the Instituto Gulbenkian de Ciência for the support in the sequencing and assembly of the Wolbachia genomes. We are thankful to the Fly Facility at Instituto Gulbenkian de Ciência for support to the Drosophila work.

\section{Author Contributions}

Conceptualization: Elves H. Duarte, Luís Teixeira.

Data curation: Elves H. Duarte, Luís Teixeira.

Formal analysis: Elves H. Duarte, Sergio López-Madrigal, Luís Teixeira.

Funding acquisition: Luís Teixeira.

Investigation: Elves H. Duarte, Ana Carvalho, Sergio López-Madrigal, João Costa.

Methodology: Elves H. Duarte, Ana Carvalho, João Costa, Luís Teixeira.

Project administration: Luís Teixeira.

Software: Elves H. Duarte.

Supervision: Luís Teixeira.

Visualization: Elves H. Duarte, Luís Teixeira.

Writing - original draft: Elves H. Duarte, Luís Teixeira.

Writing - review \& editing: Elves H. Duarte, Sergio López-Madrigal, Luís Teixeira.

\section{References}

1. Moran NA, McCutcheon JP, Nakabachi A. Genomics and Evolution of Heritable Bacterial Symbionts. Annu Rev Genet. 2008; 42: 165-190. https://doi.org/10.1146/annurev.genet.41.110306.130119 PMID: 18983256

2. Jaenike J. Coupled population dynamics of endosymbionts within and between hosts. Oikos. 2009; 118: 353-362.

3. López-Madrigal S, Duarte EH. Titer regulation in arthropod-Wolbachia symbioses. FEMS Microbiol Lett. 2019; 366: 118-9. https://doi.org/10.1093/femsle/fnz232 PMID: 31750894

4. Zug R, Hammerstein P. Still a host of hosts for wolbachia: analysis of recent data suggests that $40 \%$ of terrestrial arthropod species are infected. PLoS ONE. 2012; 7: e38544. https://doi.org/10.1371/journal. pone.0038544 PMID: 22685581

5. Werren JH, Baldo L, Clark ME. Wolbachia: master manipulators of invertebrate biology. Nat Rev Microbiol. 2008; 6: 741-751. https://doi.org/10.1038/nrmicro1969 PMID: 18794912

6. Hosokawa T, Koga R, Kikuchi Y, Meng X-Y, Fukatsu T. Wolbachia as a bacteriocyte-associated nutritional mutualist. Proc Natl Acad Sci U S A. 2010; 107: 769-774. https://doi.org/10.1073/pnas. 0911476107 PMID: 20080750

7. Hedges LM, Brownlie JC, O'Neill SL, Johnson KN. Wolbachia and virus protection in insects. Science. 2008; 322: 702. https://doi.org/10.1126/science.1162418 PMID: 18974344

8. Teixeira L, Ferreira A, Ashburner M. The bacterial symbiont Wolbachia induces resistance to RNA viral infections in Drosophila melanogaster. PLoS Biol. 2008; 6: e1000002. https://doi.org/10.1371/journal. pbio.1000002 PMID: 19222304 
9. Ross PA, Turelli M, Hoffmann AA. Evolutionary Ecology of Wolbachia Releases for Disease Control. Annu Rev Genet. 2019; 53: 93-116. https://doi.org/10.1146/annurev-genet-112618-043609 PMID: 31505135

10. Aliota MT, Walker EC, Yepes AU, Velez ID, Christensen BM, Osorio JE. The wMel Strain of Wolbachia Reduces Transmission of Chikungunya Virus in Aedes aegypti. PLoS Negl Trop Dis. 2016; 10: e0004677. https://doi.org/10.1371/journal.pntd.0004677 PMID: 27124663

11. Dutra HLC, Rocha MN, Dias FBS, Mansur SB, Caragata EP, Moreira LA. Wolbachia Blocks Currently Circulating Zika Virus Isolates in Brazilian Aedes aegypti Mosquitoes. Cell Host Microbe. 2016. https:// doi.org/10.1016/j.chom.2016.04.021 PMID: 27156023

12. Moreira LA, Iturbe-Ormaetxe I, Jeffery JA, Lu G, Pyke AT, Hedges LM, et al. A Wolbachia symbiont in Aedes aegypti limits infection with dengue, Chikungunya, and Plasmodium. Cell. 2009; 139: 12681278. https://doi.org/10.1016/j.cell.2009.11.042 PMID: 20064373

13. van den Hurk AF, Hall-Mendelin S, Pyke AT, Frentiu FD, McElroy K, Day A, et al. Impact of Wolbachia on Infection with Chikungunya and Yellow Fever Viruses in the Mosquito Vector Aedes aegypti. PLoS Negl Trop Dis. 2012; 6: e1892. https://doi.org/10.1371/journal.pntd.0001892 PMID: 23133693

14. Nazni WA, Hoffmann AA, NoorAfizah A, Cheong YL, Mancini MV, Golding N, et al. Establishment of Wolbachia Strain wAlbB in Malaysian Populations of Aedes aegypti for Dengue Control. Curr Biol. 2019; 29: 4241-4248.e5. https://doi.org/10.1016/j.cub.2019.11.007 PMID: 31761702

15. O'Neill SL, Ryan PA, Turley AP, Wilson G, Retzki K, Iturbe-Ormaetxe I, et al. Scaled deployment of Wolbachia to protect the community from Aedes transmitted arboviruses. Gates Open Res. 2018; 2: 36. https://doi.org/10.12688/gatesopenres.12844.3 PMID: 30596205

16. Chrostek E, Marialva MSP, Esteves SS, Weinert LA, Martinez J, Jiggins FM, et al. Wolbachia Variants Induce Differential Protection to Viruses in Drosophila melanogaster: A Phenotypic and Phylogenomic Analysis. PLoS Genet. 2013; 9: e1003896. https://doi.org/10.1371/journal.pgen.1003896 PMID: 24348259

17. Chrostek E, Teixeira L. Mutualism breakdown by amplification of Wolbachia genes. PLOS Biol. 2015; 13: e1002065. https://doi.org/10.1371/journal.pbio.1002065 PMID: 25668031

18. Lu P, Bian G, Pan X, Xi Z. Wolbachia Induces Density-Dependent Inhibition to Dengue Virus in Mosquito Cells. PLoS Negl Trop Dis. 2012; 6: e1754. https://doi.org/10.1371/journal.pntd.0001754 PMID: 22848774

19. Osborne SE, Leong YS, O'Neill SL, Johnson KN. Variation in antiviral protection mediated by different Wolbachia strains in Drosophila simulans. PLoS Pathog. 2009; 5: e1000656. https://doi.org/10.1371/ journal.ppat.1000656 PMID: 19911047

20. Osborne SE, Iturbe-Ormaetxe I, Brownlie JC, O'Neill SL, Johnson KN. Antiviral protection and the importance of Wolbachia density and tissue tropism in Drosophila simulans. Appl Environ Microbiol. 2012; 78: 6922-6929. https://doi.org/10.1128/AEM.01727-12 PMID: 22843518

21. McMeniman CJ, Lane RV, Cass BN, Fong AWC, Sidhu M, Wang Y-F, et al. Stable Introduction of a Life-Shortening Wolbachia Infection into the Mosquito Aedes aegypti. Science. 2009; 323: 141-144. https://doi.org/10.1126/science.1165326 PMID: 19119237

22. Min KT, Benzer S. Wolbachia, normally a symbiont of Drosophila, can be virulent, causing degeneration and early death. Proc Natl Acad Sci U S A. 1997; 94: 10792-10796. https://doi.org/10.1073/pnas.94. 20.10792 PMID: 9380712

23. McClure EE, Chávez ASO, Shaw DK, Carlyon JA, Ganta RR, Noh SM, et al. Engineering of obligate intracellular bacteria: progress, challenges and paradigms. Nat Rev Microbiol. 2017; 984: 249. https:// doi.org/10.1038/nrmicro.2017.59 PMID: 28626230

24. Ashburner M, Golic KG, Hawley RS. Drosophila: a laboratory handbook. Cold Spring Harbor, N.Y.: Cold Spring Harbor Laboratory Press; 2005.

25. Woolfit M, Iturbe-Ormaetxe I, Brownlie JC, Walker T, Riegler M, Seleznev A, et al. Genomic evolution of the pathogenic Wolbachia strain, wMelPop. Genome Biol Evol. 2013; 5: 2189-2204. https://doi.org/10. 1093/gbe/evt169 PMID: 24190075

26. Wu M, Sun LV, Vamathevan J, Riegler M, Deboy R, Brownlie JC, et al. Phylogenomics of the reproductive parasite Wolbachia pipientis wMel: a streamlined genome overrun by mobile genetic elements. PLoS Biol. 2004; 2: E69. https://doi.org/10.1371/journal.pbio.0020069 PMID: 15024419

27. Basting PJ, Bergman CM. Complete Genome Assemblies for Three Variants of the Wolbachia Endosymbiont of Drosophila melanogaster. Microbiol Res Announc. 2019; 8: 741. https://doi.org/10.1128/ MRA.00956-19 PMID: 31699757

28. Chrostek E, Teixeira L. Comment on Rohrscheib et al. 2016 "Intensity of mutualism breakdown is determined by temperature not amplification of Wolbachia genes." PLoS Pathog. 2017; 13: e1006540. https://doi.org/10.1371/journal.ppat.1006540 PMID: 28892498 
29. Christensen S, Camacho M, Sharmin Z, Momtaz AJMZ, Perez L, Navarro G, et al. Quantitative methods for assessing local and bodywide contributions to Wolbachia titer in maternal germline cells of Drosophila. BMC Microbiol. 2019; 19: 1-17. https://doi.org/10.1186/s12866-018-1372-8 PMID: 30616583

30. McGarry HF, Egerton GL, Taylor MJ. Population dynamics of Wolbachia bacterial endosymbionts in Brugia malayi. Mol Biochem Parasitol. 2004; 135: 57-67. https://doi.org/10.1016/j.molbiopara.2004.01. 006 PMID: 15287587

31. Chrostek E, Teixeira L. Within host selection for faster replicating bacterial symbionts. PLoS ONE. 2018; 13: e0191530. https://doi.org/10.1371/journal.pone.0191530 PMID: 29346449

32. Baião GC, Janice J, Galinou M, Klasson L. Comparative genomics reveals factors associated with phenotypic expression of Wolbachia. Genome Biology and Evolution 2021; evab111. https://doi.org/10. 1093/gbe/evab111 PMID: 34003269

33. Sega GA. A review of the genetic effects of ethyl methanesulfonate. Mutat Res. 1984; 134: 113-142. https://doi.org/10.1016/0165-1110(84)90007-1 PMID: 6390190

34. Grobler Y, Yun CY, Kahler DJ, Bergman CM, Lee H, Oliver B, et al. Whole genome screen reveals a novel relationship between Wolbachia levels and Drosophila host translation. PLoS Pathog. 2018; 14: e1007445. https://doi.org/10.1371/journal.ppat.1007445 PMID: 30422992

35. Burr B, Burr FA. Activation of Silent Transposable Elements. Plant Transposable Elements. Boston, MA: Springer US; 1988. pp. 317-323.

36. Duchek P, Rørth P. Guidance of Cell Migration by EGF Receptor Signaling During Drosophila Oogenesis. Science. 2001; 291: 131-133. https://doi.org/10.1126/science.291.5501.131 PMID: 11141565

37. Prelich G. Gene overexpression: uses, mechanisms, and interpretation. Genetics. 2012; 190: 841-854. https://doi.org/10.1534/genetics.111.136911 PMID: 22419077

38. Iturbe-Ormaetxe I, Burke GR, Riegler M, O'Neill SL. Distribution, expression, and motif variability of ankyrin domain genes in Wolbachia pipientis. J Bacteriol. 2005; 187: 5136-5145. https://doi.org/10. 1128/JB.187.15.5136-5145.2005 PMID: 16030207

39. Hill T, Unckless RL, Perlmutter Jl. Rapid evolution and horizontal gene transfer in the genome of a male-killing Wolbachia. bioRxiv. 2020; 2020.11.16.385294.

40. Richardson MF, Weinert LA, Welch JJ, Linheiro RS, Magwire MM, Jiggins FM, et al. Population Genomics of the Wolbachia Endosymbiont in Drosophila melanogaster. PLoS Genet. 2012; 8: e1003129. https://doi.org/10.1371/journal.pgen.1003129 PMID: 23284297

41. Reynolds KT, Thomson LJ, Hoffmann AA. The effects of host age, host nuclear background and temperature on phenotypic effects of the virulent Wolbachia strain popcorn in Drosophila melanogaster. Genetics. 2003; 164: 1027-1034. PMID: 12871912

42. Rohrscheib CE, Frentiu FD, Horn E, Ritchie FK, van Swinderen B, Weible MW, et al. Intensity of Mutualism Breakdown Is Determined by Temperature Not Amplification of Wolbachia Genes. PLoS Pathog. 2016; 12: e1005888. https://doi.org/10.1371/journal.ppat.1005888 PMID: 27661080

43. Chrostek E, Martins NE, Marialva MS, Teixeira L. Wolbachia-conferred antiviral protection is determined by developmental temperature. bioRxiv. 2020; 2020.06.24.169169. https://doi.org/10.1101/ 2020.06.24.169169

44. Ryder E, Blows F, Ashburner M, Bautista-Llacer R, Coulson D, Drummond J, et al. The DrosDel collection: a set of P-element insertions for generating custom chromosomal aberrations in Drosophila melanogaster. Genetics. 2004; 167: 797-813. https://doi.org/10.1534/genetics.104.026658 PMID: 15238529

45. Pais IS, Valente RS, Sporniak M, Teixeira L. Drosophila melanogaster establishes a species-specific mutualistic interaction with stable gut-colonizing bacteria. PLOS Biol. 2018; 16: e2005710. https://doi. org/10.1371/journal.pbio.2005710 PMID: 29975680

46. Pfaffl MW. A new mathematical model for relative quantification in real-time RT-PCR. Nucleic Acids Res. 2001; 29: e45. https://doi.org/10.1093/nar/29.9.e45 PMID: 11328886

47. Andrews, Simon. FastQC: A quality control tool for high throughput sequence data. 2010. Available: https://www.bioinformatics.babraham.ac.uk/projects/fastqc/.

48. Bolger AM, Lohse M, Usadel B. Trimmomatic: a flexible trimmer for Illumina sequence data. Bioinformatics. 2014; 30: 2114-2120. https://doi.org/10.1093/bioinformatics/btu170 PMID: 24695404

49. Li H, Durbin R. Fast and accurate short read alignment with Burrows-Wheeler transform. Bioinformatics. 2009; 25: 1754-1760. https://doi.org/10.1093/bioinformatics/btp324 PMID: 19451168

50. Li H. Minimap2: pairwise alignment for nucleotide sequences. Birol I, editor. Bioinformatics. 2018; 34 : 3094-3100. https://doi.org/10.1093/bioinformatics/bty191 PMID: 29750242

51. Wick RR, Judd LM, Gorrie CL, Holt KE. Unicycler: resolving bacterial genome assemblies from short and long sequencing reads. bioRxiv. 2016; 096412. https://doi.org/10.1101/096412 
52. Bankevich A, Nurk S, Antipov D, Gurevich AA, Dvorkin M, Kulikov AS, et al. SPAdes: A New Genome Assembly Algorithm and Its Applications to Single-Cell Sequencing. J Comput Biol. 2012; 19: 455-477. https://doi.org/10.1089/cmb.2012.0021 PMID: 22506599

53. Wick RR, Schultz MB, Zobel J, Holt KE. Bandage: interactive visualization of de novo genome assemblies. Bioinformatics. 2015; 31: 3350-3352. https://doi.org/10.1093/bioinformatics/btv383 PMID: 26099265

54. Altschul SF, Gish W, Miller W, Myers EW, Lipman DJ. Basic local alignment search tool. J Mol Biol. 1990; 215: 403-410. https://doi.org/10.1016/S0022-2836(05)80360-2 PMID: 2231712

55. Vaser R, Sovic I, Nagarajan N, Sikic M. Fast and accurate de novo genome assembly from long uncorrected reads. Genome Res. 2017; 27: 737-746. https://doi.org/10.1101/gr.214270.116 PMID: 28100585

56. Walker BJ, Abeel T, Shea T, Priest M, Abouelliel A, Sakthikumar S, et al. Pilon: An Integrated Tool for Comprehensive Microbial Variant Detection and Genome Assembly Improvement. PLoS ONE. 2014; 9: e112963. https://doi.org/10.1371/journal.pone.0112963 PMID: 25409509

57. Darling ACE, Mau B, Blattner FR, Perna NT. Mauve: multiple alignment of conserved genomic sequence with rearrangements. Genome Res. 2004; 14: 1394-1403. https://doi.org/10.1101/gr. 2289704 PMID: 15231754

58. Li H. A statistical framework for SNP calling, mutation discovery, association mapping and population genetical parameter estimation from sequencing data. Bioinformatics. 2011; 27: 2987-2993. https://doi. org/10.1093/bioinformatics/btr509 PMID: 21903627

59. Li H, Handsaker B, Wysoker A, Fennell T, Ruan J, Homer N, et al. The Sequence Alignment/Map format and SAMtools. Bioinformatics. 2009; 25: 2078-2079. https://doi.org/10.1093/bioinformatics/btp352 PMID: 19505943

60. Robinson JT, Thorvaldsdóttir H, Winckler W, Guttman M, Lander ES, Getz G, et al. Integrative genomics viewer. Nat Biotechnol. 2011; 29: 24-26. https://doi.org/10.1038/nbt.1754 PMID: 21221095

61. Team RC. R: A language and environment for statistical computing. R Foundation for Statistical Computing, Vienna, Austria; 2012. Available: URL http://www.R-project.org/.

62. Therneau TM. coxme: Mixed Effects Cox Models. 2020. Available: https://CRAN.R-project.org/ package $=$ coxme.

63. Holm S. A simple sequentially rejective multiple test procedure. Scand J Stat. 1979; 6: 65-70.

64. Burnham KP, Anderson DR. Model selection and multimodel inference: a practical information-theoretic approach. New York: Springer; 2002.

65. Duarte Elves, Carvalho A, López-Madrigal S, Costa J, Teixeira L. Data and analysis for "Forward genetics in Wolbachia: Regulation of Wolbachia proliferation by the amplification and deletion of an addictive genomic island." figshare; 2021. p. 1750726 Bytes. https://doi.org/10.6084/M9.FIGSHARE.14079920. V2 